\title{
FINITE-TIME BLOW-UP OF A NON-LOCAL STOCHASTIC PARABOLIC PROBLEM
}

\author{
NIKOS I. KAVALLARIS AND YUBIN YAN
}

\begin{abstract}
The main aim of the current work is the study of the conditions under which (finite-time) blow-up of a non-local stochastic parabolic problem occurs. We first establish the existence and uniqueness of the local-in-time weak solution for such problem. The first part of the manuscript deals with the investigation of the conditions which guarantee the occurrence of noise-induced blow-up. In the second part we first prove the $C^{1}$-spatial regularity of the solution. Then, based on this regularity result, and using a strong positivity result we derive, for first in the literature of SPDEs, a Hopf's type boundary value point lemma. The preceding results together with Kaplan's eigenfunction method are then employed to provide a (non-local) drift term induced blow-up result. In the last part of the paper, we present a method which provides an upper bound of the probability of (non-local) drift term induced blow-up.
\end{abstract}

\section{INTRODUCTION}

In the current work we consider the following non-local stochastic parabolic problem

$$
\begin{aligned}
& \frac{\partial u}{\partial t}=\Delta u+F(u)+\sigma(u) \partial_{t} W(x, t),(x, t) \in D_{T}:=D \times(0, T), \\
& u(x, t)=0, \quad(x, t) \in \Gamma_{T}:=\partial D \times(0, T), \\
& u(x, 0)=\xi(x), \quad x \in D,
\end{aligned}
$$

where $T>0$ denotes the maximum existence time and $D \subset \mathbb{R}^{d}, d \geq 1$, is a bounded domain with smooth boundary, whilst $\Delta$ denotes the Laplacian operator. Here the nonlocal reaction (drift) term $F(u)$ is defined by

$$
F(u):=\frac{\lambda f(u)}{\left(\int_{D} f(u) d x\right)^{q}}, \quad q>0,
$$

for some positive constant $\lambda$ and $f(u)$ is a locally Lipschitz and nonnegative function. The diffusion term $\sigma(u)$ is also assumed to be nonegative and Lipschitz continuous. Furthermore $\partial_{t} W(x, t)$ denotes by convention the formal time derivative of the Wiener process $\{W(x, t), x \in D, t \geq 0\}$ in a complete probability space $(\Omega, \mathcal{F}, \mathbb{P})$ with filtration $\left(\mathcal{F}_{t}\right)_{t \in[0, T]}$

Date: July 9, 2020.

1991 Mathematics Subject Classification. Primary 60H15, 35B44; Secondary 34B10 , 35B50, 35B51.

Key words and phrases. Non-local, Stochastic Partial Differential Equations, Maximum principle, Blow-up, Exponential Brownian Functionals. 
generated by $W$. The initial value $\xi$ is a $\mathcal{F}_{0}$-measurable variable in some suitable spaces introduced later.

Let $H=L^{2}(D)$ endowed with the norm $\|\cdot\|$. The solution $u(t) \equiv u(x, t ; \omega)$ of (1.1)(1.3) should be understood as an $H$-valued stochastic process on $[0, T]$ for some $T>0$ any realization $\omega \in \Omega$. Thus, questions like local-in-time existence and uniqueness of a solution of (1.1)-(1.3) arise and can be tackled with the approach developed in [15]. Questions regarding the temporal and spatial regularity of (1.1)-(1.3), which are very interesting issues, are addressed in the current work. In particular, for proving the occurrence of finite-time (non-local) drift induced blow-up we need at least a $C^{1}$-spatial regularity result, which was only quite recently obtained for the general quasilinear SPDEs, [17, and we also revive in the current work for our semilinear problem (1.1)-(1.3), cf. Theorem 4.1 .

We are strongly motivated to study problem (1.1) - (1.3) since this kind of non-local stochastic problem is associated with various industrial processes (e.g. Ohmic heating in food sterilization [35, 31, 37, 38, 52] and shear banding formation in high strain metals [5, 6, 29]) as well as with biological processes (e.g. chemotaxis phenomenon [34, 35, 55]) and statistical mechanics approaches[36], where the multiplicative noise term $\sigma(u) \partial_{t} W(x, t)$ represents the existence of external perturbations or a lack of knowledge of certain physical parameters. The occurrence of multiplicative noise terms is natural when one considers noisy control systems, see [7], and its importance is well-known in physics and biology. Many experimental or numerical observations of self-organized behavior or phase transitions arising out of such noises have been recorded in [26, 50]. For a detailed construction of a mathematical model of the form (1.1) -(1.3) arising in shear banding formation in metals the interested readers can see [29], whilst a stochastic model arising in MEMS technology is formulated and investigated in [30].

\section{State OF THE ART}

The current work mainly focuses on the phenomenon of finite-time blow-up, which in the probabilistic sense might be associated with the expectation of the solution $u$ of (1.1)(1.3) becoming infinitely big in finite time. Such a singular behaviour is definitely very interesting from the mathematical point of view, however in many applications in engineering and biology it is also correlated with some destructive behaviour of the associated mathematical models. Thus the investigation of the conditions under which such finitetime blow-up occurs becomes vital. So in the current paper we try to provide a thorough study of this issue for the non-local model (1.1) -(1.3). Before stating and proving our main results, let us review the main blow-up results available in literature. Fundamental results on the blow-up of stochastic reaction diffusion equations were first obtained by Chow ( [12, 13]) but only for the local version of problem (1.1) -(1.3), i.e. when $q=0$ in (1.4). Chow's method actually implies finite-time blow-up in the mean $L^{p}$-sense for $p>1$, see also Definition [3.7. Lv and Duan in [43], following an approach similar to [12, 13] and again for the local problem, provide a further insight on the impact of the noise term in 
the blow-up phenomenon by describing the competition between the nonlinear reaction term and noise term. Moreover, Foondun et al.[23], by extending Chow's ideas, proved the nonexistence of global solutions for the Cauchy problem, i.e. when $D=\mathbb{R}^{d}$, even for a fractional Laplacian operator. Dozzi and López-Mimbela in [20], by using a somewhat different approach, they proved a finite time blow-up result for the local problem and for a superlinear reaction term when $\sigma(u)=u$. Besides, their method also provides an upper bound of the probability of blow-up.

Thorough research has been undertaken regarding the study of finite-time blow-up of deterministic, i.e. when $\sigma \equiv 0$, reaction diffusion equations since the seminal work of Fujita [21, 22]. In particular, regarding the deterministic non-local problem

$$
\begin{aligned}
& \frac{\partial u}{\partial t}=\Delta u+\frac{\lambda f(u)}{\left(\int_{D} f(u) d x\right)^{q}}, \quad(x, t) \in D_{T}, q>0, \\
& u(x, t)=0, \quad(x, t) \in \Gamma_{T}, \\
& u(x, 0)=\xi(x), x \in D,
\end{aligned}
$$

the finite-time blow-up, i.e. the occurrence of $T<\infty$ such that

$$
\limsup _{t \rightarrow T}\|u(\cdot, t)\|_{\infty}=\infty
$$

where $\|\cdot\|_{\infty}$ denotes the norm in $L^{\infty}(D)$, has been investigated in detail in $[6$, 32, 33, 34, 37, 38, 52]. More precisely, the authors in [32] proved, that for any $0<q<1$ the solution $u$ of (2.1) - (2.3) on a convex domain $D$ blows in finite time, either for big values of the control parameter $\lambda$ or for big enough initial data $\xi(x)$, provided that $f(s)$ is a positive, increasing, convex function for any $s \in \mathbb{R}$ satisfying also the following conditions

$$
\left[f^{1-q}(s)\right]^{\prime \prime} \geq 0 \quad \text { for } \quad s \in \mathbb{R} \quad \text { and } \quad \int_{b}^{\infty} \frac{d s}{f^{1-q}(s)}<\infty, \quad \text { for any } b \in \mathbb{R} .
$$

However, to the best of our knowledge there are no any blow-up results for the stochastic non-local problem (1.1)-(1.3). Hence, the current paper initiates an investigation in this direction. Our main techniques stem from the theory of nonlinear PDEs; in particular for our investigation we basically use ideas introduced and developed in [12, 13, 20, 32.

The structure of the paper is as follows. In the first part of Section [3, we establish the existence and uniqueness of a local-in-time solution of the stochastic problem (1.1)-(1.3). The second part of Section 3 deals with the analysis of noise (diffusion) term induced blow-up. Section 4 focuses on the demonstration of the non-local reaction (drift) induced blow-up. To this end we first derive the $C^{1}$-spatial regularity for the solution $u$, and then as a by-product we prove a Hopf's type lemma for some specific stochastic problems. Notably, as far as we know it is the first time in the context of SPDEs that this key result is stated and proven. Next, making use of Hopf's lemma we derive an estimate of the solution $u$ of (1.1)-(1.3), near the boundary $\partial D$ in conjuction with the moving plane method, [3, 25, 47], adjusted in the context of SPDEs. Then the latter key estimate in conjuction with Kaplan's eigenfunction method, 28], lead to the proof of the desired 
reaction (drift) induced blow-up, which is analogous to blow-up result of the deterministic problem conisidered in [32]. Finally, Section 4 concerns with the derivation of an upper estimate of the probability of blow-up for the special case $\sigma(u)=u$, via the method introduced in [20].

\section{NOISE TERM INDUCED BLOW-UP}

In the current section we investigate the circumstances under which finite-time blow-up of the system (1.1)-(1.3) occurs due to the presence of the noise (diffusion) term. We first consider the existence and uniqueness of a weak solution locally in time by using Itô's formula and a semigroup approach. Local-in-time existence and uniqueness are rather standard and one can easilly apeals to the well known results [15]. However, due to the non-local nature of (1.1)-(1.3) and for the sake of completeness we present a detailed proof.

3.1. Existence and uniqueness of a local-in-time solution. We first set up the main functional and stochastic framework which will be used for our analysis throughout the manuscript.

Let $A=-\Delta$ with $\mathcal{D}(A)=H_{0}^{1}(D) \cap H^{2}(D)$, where $H_{0}^{1}(D)$ and $H^{2}(D)$ denote the standard Hilbert spaces and assume that $A$ has the eigenpairs $\left(\lambda_{j}, \phi_{j}\right), j=1,2,3, \ldots$ Let $L(H)$ denote the space of all bounded operators from $H=L^{2}(D)$ to $H$ and let $Q \in$ $L(H)$ be a non-negative definite and symmetric bounded operator on $H$ with orthonormal eigenfunctions $\chi_{j} \in H, j=1,2,3, \ldots$ and corresponding eigenvalues $\gamma_{j}>0, j=1,2,3, \ldots$ such that $\operatorname{Tr}(Q)=\sum_{j=1}^{\infty} \gamma_{j}<\infty$. (i.e., $Q$ is of trace class). For simplicity, hereafter we choose $\chi_{j}=\phi_{j}, j=1,2,3, \ldots$

Thereafter we let $W=W(x, t)$ denote the $H$-valued $Q$-Wiener process defined by

$$
W(x, t)=\sum_{j=1}^{\infty} \gamma_{j}^{1 / 2} \phi_{j}(x) \beta_{j}(t), \quad \text { almost surely (a.s.) }
$$

where $\beta_{j}(t)$ are independent and identically distributed $\mathcal{F}_{t}$-Brownian motions.

For the trace operator $Q \in L(H)$ with $\operatorname{Tr}(Q)<\infty$, there exists a kernel $q(x, y)$ such that

$$
(Q u)(x):=\int_{D} q(x, y) u(y) d y, \quad \text { for any } x \in D, u \in H,
$$

see [11, p. 42-43] and [42, Definition 1.64]. The kernel $q(x, y)$ is also called the covariance function of the $Q$-Wiener process $W(x, t)$.

Let $X$ be a Banach space with the norm $\|\cdot\|_{X}$. then we define the following Hilbert space

$$
L_{2}^{0}(H ; X)=\left\{\psi \in L(H, X): \sum_{j=1}^{\infty}\left\|\psi Q^{1 / 2}\left(\phi_{j}\right)\right\|_{X}^{2}=\sum_{j=1}^{\infty} \gamma_{j}\left\|\psi\left(\phi_{j}\right)\right\|_{X}^{2}<\infty\right\},
$$


with norm $\|\psi\|_{L_{2}^{0}}=\left(\sum_{j=1}^{\infty} \gamma_{j}\left\|\psi\left(\phi_{j}\right)\right\|_{X}^{2}\right)^{1 / 2} ;$ here $L(H, X)$ denotes the space of all bounded operators from $H$ to $X$. Then for any functional $\Psi:[0, T] \rightarrow L_{2}^{0}(H, X)$, the stochastic integral $\int_{0}^{T} \Psi(t) d W(t)$ is well defined, see for example [15]. For the sake of simplicity we drop the spatial dependence from $W(x, t)$ and hereafter we denote it by $W(t)$.

In order to write (1.1)-(1.3) in the abstract form, we define the Nemytskii operator

$$
F: H \rightarrow H, \quad \text { with } F(u)(x):=\frac{f(u(x))}{\left(\int_{D} f(u(x)) d x\right)^{q}}, \text { for any } x \in D \text { and } q>0 \text {, }
$$

and for any $u \in H$.

Here $f: \mathbb{R} \rightarrow \mathbb{R}$ is assumed to be a local Lipschitz continuous function, that is, for any $s_{0} \in \mathbb{R}$ there exist $\delta>0$ and $C_{f}$ such that for any $s_{1}, s_{2} \in\left\{s \in \mathbb{R}:\left|s-s_{0}\right|<\delta\right\}$ there holds

$$
\left|f\left(s_{1}\right)-f\left(s_{2}\right)\right| \leq C_{f}\left|s_{1}-s_{2}\right| .
$$

In addition, we assume that $\sigma: H \rightarrow L_{2}^{0}(H, H)$ is an $L_{2}^{0}(H, H)$-valued operator and then we may write the problem (1.1)-(1.3) as the following Itô equation in $H$,

$$
\begin{aligned}
& d u(t)=[-A u(t)+F(u(t))] d t+\sigma(u(t)) d W(t), \quad 0<t<T, \\
& u_{0}=\xi
\end{aligned}
$$

where $u(t):=u(\cdot, t)$.

We now introduce the definition of solutions of (3.4)-(3.5), see [11, 42], which will be mainly used throughout the paper.

Definition 3.1. A predictable $H$-valued stochastic process $\{u(t): t \in[0, T]\}$ is called a weak solution of (3.4) - (3.5) if for any $v \in \mathcal{D}(A)$ and almost every (a.e.) $t \in[0, T]$, the following equality holds

$$
(u(t), v)=(\xi, v)+\int_{0}^{t}[-(u(s), A v)+(F(u(s)), v)] d s+\int_{0}^{t}(\sigma(u(s)) d W(s), v),
$$

almost surely (a.s). The weak formulation (3.6) is chosen since it is more appropriate for our study on finite-time blow-up.

It is also known, [15, 42, that any weak solution $u$ of (3.4)- (3.5) is also a mild solution of (3.4) - (3.5), that is, it satisfies the following equality in $H=L^{2}(D)$,

$$
u(t)=E(t) \xi+\int_{0}^{t} E(t-s) F(u(s)) d s+\int_{0}^{t} E(t-s) \sigma(u(s)) d W(s),
$$

where $E(t)=e^{-t A}$ is the analytic semigroup generated by $-A$, see [42]. On the other hand, any regular enough mild solution is also a weak solution, cf. [15, 42].

Before proceeding with the local-in-time existence of (3.4)-(3.5) we prove the following result which will be frequently used throughout this section. 
Lemma 3.2. Assume that $f$ satisfies condition (3.3) and it is also bounded below by a positive constant, i.e. $f(s) \geq m>0, s \in \mathbb{R}$. Then the operator $F$ defined by (3.2), satisfies a locally Lipschitz condition. In particular, for any $u_{0} \in H$ there exist $\delta>0$ and $C_{F}>0$ such that for any $u_{1}, u_{2} \in B_{u_{0}, \delta}=\left\{u \in H:\left\|u-u_{0}\right\|_{\infty}<\delta\right\}$ there holds

$$
\left\|F\left(u_{1}\right)-F\left(u_{2}\right)\right\|_{H} \leq C_{F}\left\|u_{1}-u_{2}\right\|_{H} .
$$

Proof. We have

$$
\begin{aligned}
& \left|F\left(u_{1}\right)(x)-F\left(u_{2}\right)(x)\right|=\left|\lambda \frac{f\left(u_{1}(x)\right)}{\left(\int_{D} f\left(u_{1}(x)\right) d x\right)^{q}}-\lambda \frac{f\left(u_{2}(x)\right)}{\left(\int_{D} f\left(u_{2}(x)\right) d x\right)^{q}}\right| \\
& \leq \frac{\lambda\left|f\left(u_{1}(x)\right)-f\left(u_{2}(x)\right)\right|}{\left(\int_{D} f\left(u_{1}(x)\right) d x\right)^{q}} \\
& \quad+\frac{\lambda\left|f\left(u_{2}(x)\right)\right|}{\left(\int_{D} f\left(u_{1}(x)\right) d x\right)^{q}\left(\int_{D} f\left(u_{2}(x)\right) d x\right)^{q}}\left|\left(\int_{D} f\left(u_{1}(x)\right) d x\right)^{q}-\left(\int_{D} f\left(u_{2}(x)\right) d x\right)^{q}\right| \\
& \leq C_{f}(m|D|)^{-q}\left|u_{1}(x)-u_{2}(x)\right| \\
& \quad+\frac{\lambda\left|f\left(u_{2}(x)\right)\right|}{\left(\int_{D} f\left(u_{1}(x)\right) d x\right)^{q}\left(\int_{D} f\left(u_{2}(x)\right) d x\right)^{q}}\left|\left(\int_{D} f\left(u_{1}(x)\right) d x\right)^{q}-\left(\int_{D} f\left(u_{2}(x)\right) d x\right)^{q}\right| .
\end{aligned}
$$

By the mean value theorem and taking also into account (3.3), we obtain

$$
\begin{aligned}
& \left|\left(\int_{D} f\left(u_{1}(x)\right) d x\right)^{q}-\left(\int_{D} f\left(u_{2}(x)\right) d x\right)^{q}\right| \\
& =q\left(\int_{D} f(\bar{u}(x)) d x\right)^{q-1}\left|\int_{D} f\left(u_{1}(x)\right) d x-\int_{D} f\left(u_{2}(x)\right) d x\right| \\
& \leq q\left(\int_{D} f(\bar{u}(x)) d x\right)^{q-1} \int_{D}\left|f\left(u_{1}(x)\right)-f\left(u_{2}(x)\right)\right| d x \\
& \leq C_{f} q\left(\int_{D} f(\bar{u}(x)) d x\right)^{q-1} \int_{D}\left|u_{1}(x)-u_{2}(x)\right| d x \\
& \leq \widehat{C}_{f} \int_{D}\left|u_{1}(x)-u_{2}(x)\right| d x
\end{aligned}
$$

where $\bar{u}(x)$ is a value between $u_{1}(x), u_{2}(x)$. Note that if $0<q<1$ then $\widehat{C}_{f}=C_{f} q(m|D|)^{q-1}$, otherwise if $q \geq 1$ then we take $\widehat{C}_{f}=C_{f} q(M|D|)^{q-1}$ where $M=\sup _{x \in D, u \in B_{u_{0}, \delta}}\{f(u(x))\}$.

Combining (3.8) and (3.9) we finally derive, by also using Hölder's inequality,

$$
\left\|F\left(u_{1}\right)-F\left(u_{2}\right)\right\|_{H} \leq C_{F}\left\|u_{1}-u_{2}\right\|_{H}, \quad \text { whenever } \quad u_{1}, u_{2} \in B_{u_{0}, \delta} .
$$

The proof of Lemma 3.2 is now complete.

Next we establish the local-in-time existence of a weak solution to (3.4)-(3.5). 
Theorem 3.3. (Local-in-time existence) Assume that $\xi$ is $\mathcal{F}_{0}$-measurable in $H$ with $\xi \in L^{2}\left(\Omega ; L^{\infty}(D)\right)$ and (3.7) holds. Assume also that $\sigma: H \rightarrow L_{2}^{0}(H, H)$ is a locally Lipschitz continuous mapping, i.e. for any $u_{0} \in H$ there exist $\delta>0$ and $C_{\sigma}>0$ such that for any $u_{1}, u_{2} \in B_{u_{0}, \delta}=\left\{u \in H:\left\|u-u_{0}\right\|_{\infty}<\delta\right\}$ there holds

$$
\left\|\sigma\left(u_{1}\right)-\sigma\left(u_{2}\right)\right\|_{L_{2}^{0}(H, H)} \leq C_{\sigma}\left\|u_{1}-u_{2}\right\|_{H}
$$

Then the following hold true:

(1) There exists $T>0$ such that (3.4)-(3.5) has a unique weak solution $u \in L^{2}\left((0, T) ; L^{\infty}(D) \cap\right.$ $\left.W^{1,2}(D)\right) \cap L^{\infty}((0, T) ; H)$.

(2) The solution $u$ admits $H$-valued continuous trajectories and satisfies

$$
\mathbb{E}\left[\sup _{0 \leq t \leq T}\|u(t)\|_{H}^{2}\right]+\mathbb{E}\left[\int_{0}^{T}\|\nabla u(t)\|_{H}^{2} d t\right] \leq C \mathbb{E}\left[\|\xi\|_{H}^{2}\right]
$$

(3) In particular the solution $u$, seen as a stochastic process, belongs to the following functional space

$L^{2}(\Omega ; C([0, T] ; H)) \cap L^{2}\left(\Omega ; L^{2}\left((0, T) ; L^{\infty}(D) \cap W^{1,2}(D)\right)\right) \cap L^{p}\left(\Omega ; L^{\infty}\left((0, T) ; L^{p}(D)\right)\right)$, for any $p \geq 2$.

Remark 3.4. It is worth noted that due to the regularity provided by Theorem 3.3 any weak solution $u$ of (3.4)-(3.5) also satisfies for almost every (a.e.) $t \in[0, T]$ and almost surely (a.s.) the following

$$
(u(t), v)=(\xi, v)+\int_{0}^{t}[-(\nabla u(s), \nabla v)+(F(u(s)), v)] d s+\int_{0}^{t}(\sigma(u(s)) d W(s), v)
$$

for any $v \in W^{1,2}(D)$, and it is also called a variational solution of (3.4)-(3.5). Notably, all the results in the present work hold also for varational solutions of (3.4) $-(3.5)$.

A key tool for the proof of Theorem 3.3. is the following version of Itô's Lemma in Hilbert spaces.

Lemma 3.5 ([11]). Assume that $F$ and $\sigma$ satisfy (3.7) and (3.10) respectively. Assume further that $\xi$ is $\mathcal{F}_{0}$-measurable in $H$ and that $u$ satisfies the Itô process

$$
d u=(-A u+F(u)) d t+\sigma(u) d W(t), \quad u(0)=\xi .
$$

If $\psi: H \rightarrow \mathbb{R}$ is a $C^{2}(H, \mathbb{R})$ functional, then the following holds

$$
\begin{aligned}
d \psi(u(t))= & \psi^{\prime}(u(t))[(-A u(t)+F(u(t))) d t+\sigma(u(t)) d W(t)] \\
& +\frac{1}{2} \operatorname{Tr}\left(\sigma^{*}(u(t)) \psi^{\prime \prime}(u(t)) \sigma(u(t))\right) d W(t),
\end{aligned}
$$

$\sigma^{*}$ denotes the dual (transpose) operator of the diffusion operator $\sigma$. 
Proof of Theorem 3.3. The proof is inspired by [18, Theorem 3]; actually in [18] the more general quasilinear problem is tackled. In particular, here we apply the semigroup approach to establish the local-in-time existence of a mild solution for the semilinear problem (3.4)-(3.5), which is finally regular enough to be also a weak solution.

Denote

$$
S_{T}=\left\{u \mid u \in L^{2}\left(\Omega \times[0, T] ; L^{\infty}(D) \cap H_{0}^{1}(D)\right)\right\},
$$

equipped with the norm, with some suitable $\gamma>0, \delta>0$ determined later,

$$
\|u\|_{\gamma, \delta}^{2}:=\mathbb{E}\left[\int_{0}^{T} e^{-\gamma t}\left(\|u(t)\|_{H}^{2}+\delta\|\nabla u(t)\|_{H}^{2}\right) d t\right] .
$$

It is clear that $\|\cdot\|_{\gamma, \delta}$ is equivalent to $\|\cdot\|_{S_{T}}$ for any $u \in S_{T}$, where

$$
\|u\|_{S_{T}}^{2}:=\mathbb{E}\left[\int_{0}^{T}\|u(t)\|_{H_{0}^{1}(D)}^{2} d t\right] .
$$

Consider the map $\mathcal{M}: S_{T} \rightarrow L^{2}\left(\Omega \times[0, T] ; L^{\infty}(D) \cap H_{0}^{1}(D)\right)$ which is defined by

$$
\mathcal{M}(u)(t):=E(t) \xi+\int_{0}^{t} E(t-s) F(u(s)) d s+\int_{0}^{t} E(t-s) \sigma(u(s)) d W(s),
$$

where $E(t)$ is the semigroup generated by $-A$.

In the following we shall employ the Banach's fixed point theorem to prove the existence and uniqueness of a $u$ such that $\mathcal{M}(u)=u$ in $S_{T}$.

Step 1: We first show that $\mathcal{M}: S_{T} \rightarrow S_{T}$. To that end, we need to show that for any $u \in S_{T}, \mathcal{M}(u) \in S_{T}$, i.e.,

$$
\|\mathcal{M}(u)\|_{S_{T}}^{2}=\mathbb{E}\left[\int_{0}^{T}\|\mathcal{M}(u)(t)\|_{H_{2}^{1}(D)}^{2} d t\right]<\infty,
$$

which actually follows by the assumptions on $\xi, F$ and $\sigma$.

Step 2: Next we show that $\mathcal{M}$ is a contaction operator, i.e. there exist positive constants $\gamma, \delta$ and $0<\kappa<1$ such that

$$
\|\mathcal{M}(u)-\mathcal{M}(v)\|_{\gamma, \delta} \leq \kappa\|u-v\|_{\gamma, \delta},
$$

where $\kappa=\kappa(F, \sigma)$ depends on $F$ and $\sigma$.

In fact, by (3.12) we have

$$
\begin{aligned}
\bar{u}(t):=\mathcal{M}(u)(t)-\mathcal{M}(v)(t)= & \int_{0}^{t} E(t-s)(F(u(s))-F(v(s))) d s \\
& +\int_{0}^{t} E(t-s)(\sigma(u(s))-\sigma(v(s))) d W(s),
\end{aligned}
$$

which satisfies the Itô problem

$$
d \bar{u}+A \bar{u} d t=[F(u)-F(v)] d t+[\sigma(u)-\sigma(v)] d W(t) .
$$


Let $w(t)=\bar{u}(t) e^{-\frac{\gamma}{2} t}$, then $w(t)$ satisfies

$$
d w+A w d t=-\frac{\gamma}{2} w d t+[F(u)-F(v)] e^{-\frac{\gamma}{2} t} d t+[\sigma(u)-\sigma(v)] e^{-\frac{\gamma}{2} t} d W(t) .
$$

Implementing Itô's formula, see Lemma 3.5, with $\varphi(w)=\|w\|_{H}^{2}$ we deduce

$$
\begin{aligned}
& e^{-\gamma T}\|\bar{u}(T)\|_{H}^{2}+2 \int_{0}^{T} e^{-\gamma t}\|\nabla \bar{u}(t)\|_{H}^{2} d t \\
& =-\gamma \int_{0}^{T} e^{-\gamma t}\|\bar{u}(t)\|_{H}^{2} d t+2 \int_{0}^{T} e^{-\gamma t}(\bar{u}(t), F(u(t))-F(v(t))) d t \\
& +\int_{0}^{T} e^{-\gamma t}\|\sigma(u(t))-\sigma(v(t))\|_{L_{2}^{0}(H, H)}^{2} d t .
\end{aligned}
$$

Notably for any small $\epsilon>0$, and by virtue of Young's inequality, we obtain some constant $C_{\epsilon}$ depending on $\epsilon$ such that

$$
\begin{aligned}
& 2 \int_{0}^{T} e^{-\gamma t}(\bar{u}(t), F(u(t))-F(v(t))) d t \\
& \leq \epsilon \int_{0}^{T} e^{-\gamma t}\|F(u(t))-F(v(t))\|_{H}^{2} d t+C_{\epsilon} \int_{0}^{T} e^{-\gamma t}\|\bar{u}(t)\|_{H}^{2} d t \\
& \leq \epsilon C_{F} \int_{0}^{T} e^{-\gamma t}\|u(t)-v(t)\|_{H}^{2} d t+C_{\epsilon} \int_{0}^{T} e^{-\gamma t}\|\bar{u}(t)\|_{H}^{2} d t,
\end{aligned}
$$

taking also into account that $F$ satisfies a locally Lipschitz condition with constant $C_{F}$ by Lemma 3.2 .

Furthermore, due to (3.10) we have

$$
\int_{0}^{T} e^{-\gamma t}\|\sigma(u(t))-\sigma(v(t))\|_{L_{2}^{0}(H, H)}^{2} d t \leq C_{\sigma} \int_{0}^{T} e^{-\gamma t}\|u(t)-v(t)\|_{H}^{2} d t
$$

and thus by virtue of (3.13) we obtain

$$
\begin{aligned}
& e^{-\gamma T}\|\bar{u}(T)\|_{H}^{2}+2 \int_{0}^{T} e^{-\gamma t}\|\nabla \bar{u}(t)\|_{H}^{2} d t+\left(\gamma-C_{\epsilon}\right) \int_{0}^{T} e^{-\gamma t}\|\bar{u}(t)\|_{H}^{2} d t \\
& \leq\left(\epsilon C_{F}+C_{\sigma}\right) \int_{0}^{T} e^{-\gamma t}\|u(t)-v(t)\|_{H}^{2} d t .
\end{aligned}
$$

Taking the expectation on both sides of (3.14), noting also that $\mathbb{E}\left[e^{-\gamma T}\|\bar{u}(T)\|_{H}^{2}\right] \geq 0$, we derive

$$
\begin{aligned}
& \left(\gamma-C_{\epsilon}\right) \mathbb{E}\left[\int_{0}^{T} e^{-\gamma t}\|\bar{u}(t)\|_{H}^{2} d t\right]+2 \mathbb{E}\left[\int_{0}^{T} e^{-\gamma t}\|\nabla \bar{u}(t)\|_{H}^{2} d t\right] \\
& \leq \epsilon C_{F} \mathbb{E}\left[\int_{0}^{T} e^{-\gamma t}\|u(t)-v(t)\|_{H}^{2} d t\right]+C_{\sigma} \mathbb{E}\left[\int_{0}^{T} e^{-\gamma t}\|u(t)-v(t)\|_{H}^{2} d t\right]
\end{aligned}
$$


or equivalently

$$
\begin{aligned}
& \mathbb{E}\left[\int_{0}^{T} e^{-\gamma t}\|\bar{u}(t)\|_{H}^{2} d t\right]+\frac{2}{\gamma-C_{\epsilon}} \mathbb{E}\left[\int_{0}^{T} e^{-\gamma t}\|\nabla \bar{u}(t)\|_{H}^{2} d t\right] \\
& \leq \frac{\epsilon C_{F}+C_{\sigma}}{\gamma-C_{\epsilon}} \mathbb{E}\left[\int_{0}^{T} e^{-\gamma t}\|u(t)-v(t)\|_{H}^{2} d t\right],
\end{aligned}
$$

provided $\gamma>C_{\epsilon}$.

Choosing now $\gamma$ sufficiently large and suitable $\epsilon>0$ such that $0<\frac{\epsilon C_{F}+C_{\sigma}}{\gamma-C_{\epsilon}}<\kappa<1$ we have

$$
\begin{aligned}
& \mathbb{E}\left[\int_{0}^{T} e^{-\gamma t}\left(\|\bar{u}(t)\|_{H}^{2}+\delta\|\nabla \bar{u}(t)\|_{H}^{2}\right) d t\right] \\
& \leq \kappa \mathbb{E}\left[\int_{0}^{T} e^{-\gamma t}\left(\|u(t)-v(t)\|_{H}^{2}+\delta\|\nabla(u(t)-v(t))\|_{H}^{2}\right) d t\right]
\end{aligned}
$$

for $\delta=\frac{2}{\gamma-C_{\epsilon}}$. The latter entails

$$
\|\mathcal{M}(u)-\mathcal{M}(v)\|_{\gamma, \delta} \leq \kappa\|u-v\|_{\gamma, \delta}, \quad 0<\kappa<1,
$$

and thus by Banach's fixed point theorem, there exists a unique local-in-time solution $u \in S_{T}$ for the problem (3.4)-(3.5). Finally, the estimate (3.11) can be obtained by following a similar argument as in the proof of Theorem 3 in [18].

Step 3: Finally we show that $u \in L^{p}\left(\Omega ; L^{\infty}\left((0, T) ; L^{p}(D)\right)\right), p \geq 2$. Note that $f$ : $\mathbb{R} \rightarrow \mathbb{R}$ satisfies a local Lipschitz condition and hence in conjunction with Lemma 3.2 we actually get

$$
\|F(u(t))-F(v(t))\|_{L^{p}(D)} \leq C_{F}\|u(t)-v(t)\|_{L^{p}(D)},
$$

and

$$
\|\sigma(u(t))-\sigma(v(t))\|_{L_{2}^{0}\left(H, L^{p}(D)\right)} \leq C_{\sigma}\|u(t)-v(t)\|_{L^{p}(D)} .
$$

Thus we may again use the same arguments as in Steps 1 and 2 to show

$$
u \in L^{p}\left(\Omega ; L^{\infty}\left((0, T) ; L^{p}(D)\right)\right), \quad \text { for } \quad p \geq 2,
$$

and so the mild solution derived in Step 2 is also a weak solution, cf. [15, 42]. The proof of Theorem 3.3 is now complete.

Remark 3.6. If we consider initial data $\xi(x) \geq 0$ almost surely (a.s) then our local solution $u(x, t)$ is also positive a.s by application of the comparison principle, see [10].

3.2. Noise term induced finite-time blow-up. In this subsection we investigate the impact of the noise term on the phenomenon of finite-time blow-up. We actually prove that the finite-time blow-up occurs, when the noise term is so big that it dominates the drift term and thus leads the dynamics of the stochastic system.

Before proceeding further with the mathematical analysis, we first define the notion of finite-time blow-up for problem (3.4)-(3.5). 


\section{Definition 3.7. (Finite-time blow-up)}

The solution $u$ of problem (3.4)-(3.5) (or equivalently that of (1.1)-(1.3)) blows up in finite time in the sense of mean $L^{p}$-norm if there exists $0 \leq T^{*}<\infty$ such that

$$
\limsup _{t \rightarrow T^{*}} \mathbb{E}\left[\|u\|_{p}\right]=\infty
$$

for some $1 \leq p \leq \infty$. Here $\|\cdot\|_{p}$ denotes the norm in $L^{p}(D)$.

Throughout this subsection we assume the following:

$\left(S_{1}\right)$ The correlation function $q(x, y)$ of the Wiener process $W(t)$ is continuous and positive for any $x, y \in \bar{D}$ and satisfies

$$
\int_{D} \int_{D} q(x, y) w(x) w(y) d x d y \geq q_{1} \int_{D} w^{2}(x) d x,
$$

for any positive $w \in H$ and for some $q_{1}>0$. This actually means that the correlation function behaves as a steep Gaussian function.

$\left(S_{2}\right) \sigma(s)$ is a convex function and there also exists a positive, strictly increasing, convex and superlinear function $G_{1}(s)$ such that

$$
\sigma^{2}(s) \geq 2 G_{1}\left(s^{2}\right) \quad \text { for } \quad s \geq 0 \text { and } \quad \int_{0}^{\infty} \frac{d s}{G_{1}(s)}<\infty .
$$

We also consider as $\left(\lambda_{1}, \phi_{1}\right)$ the first eigenpair of the operator $A=-\Delta: \mathcal{D}(A)=H_{0}^{1}(D) \cap$ $W^{2,2}(D)$, i.e. there holds

$$
-\Delta \phi_{1}=\lambda_{1} \phi_{1}, x \in D \quad \text { and } \quad \phi_{1}=0, x \in \partial D .
$$

It is known that $\phi_{1}$, see [16], has a constant sign on $D$ so we can take $\phi_{1} \geq 0$ on $\bar{D}$ and it can be also normalized so that

$$
\int_{D} \phi_{1} d x=1
$$

Next following the approach of [12] we obtain the following.

Theorem 3.8. Under conditions $\left(S_{1}\right)$ and $\left(S_{2}\right)$ the (unique) local-in-time solution $u$ to (3.4) -(3.5) (or equivalently to (1.1) -(1.3) ), and provided by Theorem 3.3, blows up in finite time if the initial data $\xi \in L^{2}(\Omega ; H)$ satisfy $\xi(x) \geq 0$ a.s. and

$$
\theta(0)=\theta_{0}=: \mathbb{E}\left[\left(\int_{D} \xi(x) \phi_{1}(x) d x\right)^{2}\right]>\gamma,
$$

where $\gamma$ is the largest root of the equation $\beta(s):=2 \widehat{q}_{1} G_{1}(s)-2 \lambda_{1} s=0$ and $\widehat{q}_{1}$ is some positive constant.

Proof. We first define

$$
\hat{u}(t):=\int_{D} u(x, t) \phi_{1}(x) d x .
$$


Then we take $v=\phi_{1}$ as a test function into weak formulation (3.6) to deduce

$$
\begin{aligned}
\hat{u}(t):=\int_{D} u(x, t) \phi_{1}(x) d x & =\int_{D} \xi(x) \phi_{1}(x) d x-\int_{0}^{t} \int_{D} u(x, s)\left(A \phi_{1}\right)(x) d x d s \\
& +\lambda \int_{0}^{t} \int_{D} \frac{f(u(x, s)) \phi_{1}(x)}{\left(\int_{D} f(u(x, s)) d x\right)^{q}} d x d s \\
& +\int_{0}^{t} \int_{D} \sigma(u(x, s)) \phi_{1}(x) d x d W(x, s) \\
& =\int_{D} \xi(x) \phi_{1}(x) d x-\lambda_{1} \int_{0}^{t} \int_{D} u(x, s) \phi_{1}(x) d x d s \\
& +\lambda \int_{0}^{t} \int_{D} \frac{f(u(x, s)) \phi_{1}(x)}{\left(\int_{D} f(u(x, s)) d x\right)^{q}} d x d s \\
& +\int_{0}^{t} \int_{D} \sigma(u(x, s)) \phi_{1}(x) d x d W(x, s) .
\end{aligned}
$$

Next Itô's formula, i.e. Lemma 3.5, for $\psi(u)=u^{2}$ implies

$$
\begin{aligned}
\hat{u}^{2}(t) & =\left(\int_{D} \xi(x) \phi_{1}(x) d x\right)^{2}-2 \lambda_{1} \int_{0}^{t} \hat{u}^{2}(s) d s \\
& +2 \lambda \int_{0}^{t} \int_{D} \hat{u}(s) \frac{f(u(x, s)) \phi_{1}(x)}{\left(\int_{D} f(u(x, s)) d x\right)^{q}} d x d s+2 \int_{0}^{t} \int_{D} \hat{u}(s) \sigma(u(x, s)) \phi_{1}(x) d x d W(x, s) \\
& +\int_{0}^{t} \int_{D} \int_{D} q(x, y) \phi_{1}(x) \phi_{1}(y) \sigma(u(x, s)) \sigma(u(y, s)) d x d y d s
\end{aligned}
$$

where (3.17) is also taking into account.

Set $\theta(t):=\mathbb{E}\left[\hat{u}^{2}(t)\right]$, then by taking the expectation into (3.18) and interchanging the order of expectation and integration by virtue of Fubini's theorem, we have

$$
\begin{aligned}
\theta(t) & =\mathbb{E}\left[\left(\int_{D} \xi(x) \phi_{1}(x) d x\right)^{2}\right]-2 \lambda_{1} \int_{0}^{t} \theta(s) d s \\
& +2 \mathbb{E}\left[\int_{0}^{t} \int_{D} \hat{u}(s) \frac{f(u(x, s)) \phi_{1}(x)}{\left(\int_{D} f(u(x, s)) d x\right)^{q}} d x d s\right] \\
& +\mathbb{E}\left[\int_{0}^{t} \int_{D} \int_{D} q(x, y) \phi_{1}(x) \phi_{1}(y) \sigma(u(x, s)) \sigma(u(y, s)) d x d y d s\right]
\end{aligned}
$$

where we use the following result

$$
\mathbb{E}\left[\int_{0}^{t} \int_{D} \hat{u}(s) \sigma(u(x, s)) \phi_{1}(x) d x d W(x, s)\right]=0 .
$$


Alternatively (3.19) can be written in the differential form

$$
\begin{aligned}
\frac{d \theta}{d t}=\quad & -2 \lambda_{1} \theta(t)+2 \lambda \mathbb{E}\left[\hat{u}(t) \int_{D} \frac{f(u(x, t)) \phi_{1}(x)}{\left(\int_{D} f(u(x, t)) d x\right)^{q}} d x\right] \\
+ & \mathbb{E}\left[\int_{D} \int_{D} q(x, y) \phi_{1}(x) \phi_{1}(y) \sigma(u(x, t)) \sigma(u(y, t)) d x d y\right], t>0,
\end{aligned}
$$

with initial condition

$$
\theta(0)=\theta_{0}:=\mathbb{E}\left[\left(\int_{D} \xi(x) \phi_{1}(x) d x\right)^{2}\right] .
$$

Now assumptions $\left(S_{1}\right)$ and $\left(S_{2}\right)$ along with Jensen's and Hölder's inequalities imply that the third term in the right side of (3.20) is estimated as

$$
\begin{aligned}
& \mathbb{E}\left[\int_{D} \int_{D} q(x, y) \phi_{1}(x) \phi_{1}(y) \sigma(u(x, t)) \sigma(u(y, t)) d x d y\right] \\
& \geq q_{1} \mathbb{E}\left[\int_{D} \phi_{1}^{2}(x) \sigma^{2}(u(x, t)) d x\right] \geq \tilde{q}_{1} \mathbb{E}\left[\int_{D} \phi_{1}(x) \sigma(u(x, t)) d x\right]^{2} \\
& \geq \tilde{q}_{1} \mathbb{E}\left[\sigma^{2}(\hat{u}(t))\right] \geq 2 \tilde{q}_{1} \mathbb{E}\left[G_{1}\left(\hat{u}^{2}(t)\right)\right] \geq 2 \widehat{q}_{1} G_{1}(\theta(t)),
\end{aligned}
$$

for some appropriate positive constant $\widehat{q}_{1}$, where (3.16) has been also taken into consideration.

Therefore $\theta$ satisfies

$$
\begin{aligned}
& \frac{d \theta(t)}{d t} \geq-2 \lambda_{1} \theta(t)+2 \widehat{q}_{1} G_{1}(\theta(t)):=\beta(\theta(t)), t>0, \\
& \theta(0)=\mathbb{E}\left[\left(\xi, \phi_{1}\right)^{2}\right],
\end{aligned}
$$

using also the fact that the second term in (3.20) is positive, see also Remark 3.6.

Let now $\gamma$ be the largest root of the equation $\beta(s)=0$, then we have $\beta(s)>0$ for any $s>\gamma$ if $\gamma>0$. Otherwise, if $\gamma=0$ then we have $\beta(s)>0$ for any $s>0$. Therefore, if we take $\theta(0)>\gamma$ then by (3.21) $-(3.22)$ we have

$$
t \leq \int_{\theta_{0}}^{\theta(t)} \frac{d s}{\beta(s)} \leq \int_{\theta_{0}}^{\infty} \frac{d s}{\beta(s)}
$$

Next using that $G_{1}(s)$ is a superlinear function, due to (3.15), we derive

$$
t \leq \int_{\theta_{0}}^{\theta(t)} \frac{d s}{\beta(s)} \leq \int_{\theta_{0}}^{\infty} \frac{d s}{\beta(s)} \leq \frac{1}{N} \int_{\theta_{0}}^{\infty} \frac{d s}{G_{1}(s)}<\infty
$$

for some positive constant $N$, hence

$$
\theta(t) \rightarrow \infty \text { as } t \rightarrow T_{1},
$$


where

$$
T_{1} \leq \int_{\theta_{0}}^{\infty} \frac{d s}{G_{1}(s)}<\infty .
$$

Notably, by virtue of Hölder's inequality we derive

$$
\theta(t):=\mathbb{E}\left[\hat{u}^{2}(t)\right] \leq \mathbb{E}\left[\|u\|_{2}^{2}\right],
$$

which in conjunction with (3.23) implies

$$
\mathbb{E}\left[\|u\|_{2}^{2}\right] \rightarrow \infty \text { as } t \rightarrow T^{*} \leq T_{1} .
$$

The proof of the Theorem is now complete.

Remark 3.9. Since $u$ is bounded in $D \times[0, T)$ then (3.16) and (3.24), via Theorem [3.3, imply

$$
\theta(t):=\mathbb{E}\left[\hat{u}^{2}(t)\right] \leq \mathbb{E}\left[\|u\|_{\infty}^{2}\right] \rightarrow \infty, \quad \text { as } t \rightarrow T_{b} \leq T_{1} .
$$

Consequently Theorem 3.8 entails the finite-time blow-up of the stochastic process $u$ with respect to $L^{\infty}$ - norm as well as according to Definition 3.7.

Remark 3.10. The result of Theorem 3.8 with $f(s)=e^{s}$ and $q>1$ complements the results of Theorems 4.1 and $4.2 \mathrm{in}$ [4. Indeed those theorems state that when $\sigma(s)=0$, i.e. for the deterministic case, only a global-in-time solution exists. Consequently, Theorem 3.8 unveils that a dominant noise can change dramatically the dynamical behaviour of the solution leading to finite-time blow-up. Moreover Theorem 3.8 ensures the occurrence of finite-time blow-up in the case $f(s)=e^{s}, q=1$, for any dimension $d>2$, a result that was only conjectured for the deterministic case and only proven for $d=2$, see in [34]. In the latter case problem (1.1)-(1.3) is stochastic perturbation of a problem which describes the biological phenomenon of chemotaxis and so the occurrence of finite-time blow-up describes the aggregation of a biological population.

\section{DRIFT TERM INDUCED BLOW-UP}

This section deals with the finite-time blow-up of (1.1)-(1.3) induced by the non-local drift (reaction) term. For the proof of such results a delicate estimate of the non-local term is needed, which actually arises as a by-product of an estimate of the solution $u$ of (1.1) - (1.3) near the boundary $\partial D$. The control of $u$ near the boundary is obtained via the moving plane method, which requires the validity of a Hopf's type result for the stochastic problem (1.1)-(1.3) . However, for such a result to be proven the $C^{1}$-spatial regularity of $u$ is necessary which is established below.

For the purposes of the current section the positive nonlinearity $f(s)$ is assumed to be increasing and convex, i.e.

$$
f^{\prime}(s), f^{\prime \prime}(s) \geq 0 \quad \text { for } \quad s \in \mathbb{R} \text {. }
$$


4.1. Spatial regularity of the solutions of (1.1)-(1.3). In the sequel, by following the approach introduced in [17], we prove the spatial $C^{1}$-spatial regularity of the solutions of (1.1)-(1.3). Such a result will be used to derive the desired control of the solution near the boundary. Before we proceed with the proof we introduce the required functional framework.

Let $C^{\bar{\alpha}, \bar{\beta}}(\bar{D} \times[0, T]), 0<\bar{\alpha} \leq 1,0<\bar{\beta} \leq 1$ denote the Hölder spaces equipped with the norm

$$
\|g\|_{C^{\bar{\alpha}, \bar{\beta}}}=\sup _{(x, t)}|g(x, t)|+\sup _{(x, t) \neq(y, s)} \frac{|g(x, t)-g(y, s)|}{|x-y|^{\bar{\beta}}+|t-s|^{\bar{\alpha}}} .
$$

With usual modifications, we can also consider the case for $\bar{\alpha}, \bar{\beta} \geq 1$. Note that it holds

$$
C^{\bar{\alpha}}\left([0, T] ; C^{\bar{\beta}}(\bar{D})\right) \nsubseteq C^{\bar{\alpha}, \bar{\beta}}(\bar{D} \times[0, T]),
$$

and therefore we have to distinguish these two spaces.

Let, for any $p>1, r \geq 0$,

$$
H^{r, p}(D)=\left\{h \mid\|h\|_{H^{r, p}(D)}:=\inf \left\{\|g\|_{H^{r, p}\left(\mathbb{R}^{d}\right)},\left.g\right|_{D}=h\right\}<\infty\right\},
$$

where, the so called Bessel potential space, is defined as

$$
H^{r, p}\left(\mathbb{R}^{d}\right)=\left\{h \mid(I-\Delta)^{r / 2} h \in L^{p}\left(\mathbb{R}^{d}\right),\right\}
$$

where

$$
(I-\Delta)^{r / 2} h:=\mathcal{F}^{-1}\left(\left(1+|\xi|^{2}\right)^{r / 2} \hat{h}\right) .
$$

Here $\hat{h}$ denotes the Fourier transform of $h$, i.e., $\hat{h}=\mathcal{F}(h)$, and $\mathcal{F}^{-1}$ denotes the inverse Fourier transform. The choice of this scale of function spaces is more natural for our method than the standard Sobolev spaces $W^{r, p}(D), p>1, r \geq 0$, cf. [17]. The spaces $H^{r, p}(D)$ are generally different from the Sobolev spaces $W^{r, p}(D)$. However, the two following cases can occur

$$
W^{r, p}(D)=H^{r, p}(D) \quad \text { if } \quad r \in \mathbb{N}_{0}, p \in[1, \infty) \quad \text { or } \quad r \geq 0, p=2,
$$

and

$$
H^{r+\varepsilon, p}(D) \hookrightarrow W^{r, p}(D) \hookrightarrow H^{r-\varepsilon, p}(D), r \in \mathbb{R}, p \in(1, \infty), \varepsilon>0 .
$$

Furthermore, if $D$ is sufficiently regular, as in our case, then $H^{r, p}(D)$ coincides with the space of restrictions of functions in $H^{r, p}\left(\mathbb{R}^{d}\right)$ to $D$ and thus the Sobolev embedding theorem holds true. Then the spaces $H_{0}^{r, p}(D), r \geq 0, p \in(1, \infty)$, are defined as the closure of $C_{c}^{\infty}(D)$ in $H^{r, p}(D)$. Note that $H_{0}^{r, p}(D)=H^{r, p}(D)$ whenever $r \leq 1 / p$, while $H_{0}^{r, p}(D)$ is strictly contained in $H^{r, p}(D)$ if $r>1 / p$.

Finally, it is worth noting that the Bessel potential spaces $H^{r, p}(D), p \geq 2, r>0$ are well suited for the stochastic Itô integration (see [9] for the precise construction of the stochastic integral). 
Notably, in order to obtain the desired $C^{1}$-spatial regularity for the solution of (1.1)(1.3), we need some further restrictions on the diffusion operator $\sigma$. Indeed, we consider the following assumption:

$(\sigma) \quad \sigma: H \rightarrow L_{2}^{0}\left(H, H^{r, p}(D)\right)$ satisfies the linear growth condition, i.e.,

$$
\|\sigma(u)\|_{L_{2}^{0}\left(H, H^{r, p}(D)\right)} \leq C\left(1+\|u\|_{H^{r, p}(D)}\right), \quad \text { for } p \geq 2, \quad \text { and } \quad 0 \leq r \leq 1 .
$$

Then the following regularity result can be proved by using the approach demonstrated in [17, Proposition 5.1]. For readers' convenience and for the sake of completeness we provide below a complete proof adjusted to the stochastic problem (3.4)-(3.5).

Theorem 4.1. ( $C^{1}$-spatial regularity ) Let us consider that all assumptions of Theorem 3.3 hold true. Further assume that the condition $(\sigma)$ holds and that $f$ satisfies (3.3). If $\xi \in L^{m}\left(\Omega ; C^{1+l}(\bar{D})\right)$, for $m \geq 2, l>0$, then for all $\alpha \in(0,1 / 2)$, there exists $\beta>0$ such that

$$
u \in L^{m}\left(\Omega ; C^{1+\beta, \alpha}(\bar{D} \times[0, T])\right), \quad \text { for any } m \geq 2 .
$$

Proof. We first show that, there exists $\eta>0$ such that

$$
u \in L^{m}\left(\Omega ; C^{\eta}(\bar{D} \times[0, T])\right), \quad \text { for any } m \geq 2 .
$$

Set $u=y+z$, where $z$ solves the following linear SPDE

$$
\begin{aligned}
& d z=-A z d t+\sigma(u) d W(t), 0<t<T, \\
& z(0)=0,
\end{aligned}
$$

whilst $y$ is the unique solution of the linear deterministic PDE problem

$$
\begin{aligned}
& \frac{d y}{d t}=-A y+F(u), 0<t<T, \\
& y(0)=\xi .
\end{aligned}
$$

Step 1. Hölder regularity of z. By Theorem 3.3, the weak solution $u$ of (3.4)-(3.5) belongs to $L^{m}\left(\Omega ; L^{m}\left((0, T) ; L^{m}(D)\right)\right), m \geq 2$. Then the assumption $(\sigma)$ with $r=0$ implies that $\sigma(u)$ belongs to $L^{m}\left(\Omega ; L^{m}\left((0, T) ; L_{2}^{0}\left(H, L^{m}(D)\right)\right)\right)$. Hence the Hölder's regularity for the stochastic integral

$$
z=\int_{0}^{t} E(t-s) \sigma(u(s)) d W(s)
$$

is easily obtained. Indeed, using the linear growth of $\sigma$ and the factorization method, see [9. Corollary 3.5], we have

$$
\mathbb{E}\left[\|z\|_{C^{\gamma}\left([0, T] ; H_{0}^{\delta, m}(D)\right)}^{m}\right] \leq C\left(1+\mathbb{E}\|u\|_{L^{m}\left((0, T) ; L^{m}(D)\right)}^{m}\right),
$$

where $\gamma \in\left[0, \frac{1}{2}-\frac{1}{m}-\frac{\delta}{2}\right), \quad \delta \in\left(0,1-\frac{2}{m}\right), m>2$. Now assume that $m \geq 3$, then $\delta=\frac{1}{6}, \gamma=\frac{1}{12}$ satisfy the conditions above uniformly. Choose $m \geq m_{0}=7 d$, where $d$ is 
the spatial dimension and also take $\alpha=\delta-\frac{d}{m_{0}}$, then by Sobolev's embedding theorem, we have

$$
H^{\delta, m}(D) \hookrightarrow C^{\alpha}(D),
$$

since $\delta-\frac{d}{m}>\delta-\frac{d}{m_{0}}=\alpha$. Thus for any $m \geq m_{0}$,

$$
\mathbb{E}\left[\|z\|_{C^{\gamma}\left([0, T] ; C^{\alpha}(D)\right)}^{m}\right] \leq C\left(1+\mathbb{E}\left[\|u\|_{L^{m}\left((0, T) ; L^{m}(D)\right)}^{m}\right]\right)<\infty .
$$

On the other hand, for $m \in\left[2, m_{0}\right)$, we have

$$
\mathbb{E}\left[\|z\|_{C^{\gamma}\left([0, T] ; C^{\alpha}(D)\right)}^{m}\right] \leq\left(\mathbb{E}\left[\|z\|_{C^{\gamma}\left([0, T] ; C^{\alpha}(D)\right)}^{m_{0}}\right]\right)^{m / m_{0}}<\infty .
$$

Thus for any $m \geq 2$, we have

$$
\mathbb{E}\left[\|z\|_{C^{\gamma}\left([0, T] ; C^{\alpha}(D)\right)}^{m}\right]<\infty .
$$

Step 2. Hölder regularity of $y$. Due to Lemma 3.2, the functional $F$ satisfies a locally Lipschitz condition and hence the following estimate is valid

$$
\mathbb{E}\left[\|F(u)\|_{L^{r}\left((0, T) ; L^{r}(D)\right)}^{r}\right] \leq C\left(1+\mathbb{E}\left[\|u\|_{L^{r}\left((0, T) ; L^{r}(D)\right)}^{r}\right]\right)<\infty,
$$

for any $r \geq 2$ by virtue of Theorem 3.3 .

Now choosing $r \geq 2$ such that $\frac{2+d}{r}<\frac{1}{2}$, we have by classical parabolic PDE theory (see Theorems 7.1 and 10.1 in [40]),

$$
\|y\|_{C^{\alpha, \alpha / 2}(\bar{D} \times[0, T])} \leq C\left(1+\|\xi\|_{C^{l}(\bar{D})}\right)\left(1+\|F(u)\|_{L^{r}\left((0, T) ; L^{r}(D)\right)}^{2 d+1}\right), \quad r \geq 2,
$$

for some $\alpha>0$ and thus

$$
\|y\|_{C^{\alpha, \alpha / 2}(\bar{D} \times[0, T])}^{m} \leq C\left(1+\|\xi\|_{C^{l}(\bar{D})}^{2 m}\right)\left(1+\|F(u)\|_{L^{r}\left((0, T) ; L^{r}(D)\right)}^{r}\right),
$$

provided that $2(2 d+1) m<r$.

Since $r$ is arbitrary in $[2, \infty)$, then (4.5) implies that

$$
\mathbb{E}\left[\|y\|_{C^{\alpha, \alpha / 2}(\bar{D} \times[0, T])}^{m}\right]<\infty, \quad \text { for any } m \in[2, \infty) .
$$

Choose now $\eta=\min \left\{\frac{\alpha}{2}, \gamma, \lambda\right\}>0$, then taking into account (4.4) and (4.6) we derive estimate (4.3).

Step 3. Higher spatial Hölder regularity of $z$. Given estimate (4.3) and using also Sobolev's embedding theorem we conclude that $u \in L^{m}\left(\Omega ; L^{m}\left((0, T) ; H^{k, m}(D)\right)\right)$ for $k<\eta<1 / 2$, hence by the assumption $(\sigma)$, we have

$$
\sigma(u) \in L^{m}\left(\Omega ; L^{m}\left((0, T) ; L_{2}^{0}\left(H, H^{k, m}(D)\right)\right)\right) .
$$

Using again the factorization method [9, Corollary 3.5], we obtain

$$
\mathbb{E}\left[\|z\|_{C^{\gamma}\left([0, T] ; H^{\delta+k, m}(D)\right)}^{m}\right] \leq C\left(1+\mathbb{E}\left[\|u\|_{L^{m}\left((0, T) ; H^{k, m}(D)\right)}^{m}\right]\right),
$$

where $\gamma \in\left[0, \frac{1}{2}-\frac{1}{m}-\frac{\delta}{2}\right)$ and $\delta \in\left(0,1-\frac{2}{m}\right)$ for any $m>2$. In the sequel we assume $m \geq m_{0}:=(d+4) / k$ and thus $\delta=1-3 / m_{0}$ and $\gamma=1 /\left(4 m_{0}\right)$ satisfy the conditions 
above uniformly in $m \geq m_{0}$. Notably we have that $(\delta+k) m>k m \geq k m_{0} \geq d$ and thus the following Sobolev embedding holds true:

$$
H^{\delta+k, m}(D) \hookrightarrow C^{\theta}(D), \text { for } \theta=k+\delta-d / m_{0} .
$$

Moreover via the definition of $\delta$ we have $\theta=k+1-\frac{d+3}{m_{0}}>1$.

Therefore, we finally deduce

$$
\mathbb{E}\left[\|z\|_{C^{\gamma}\left([0, T] ; C^{\theta}(D)\right)}^{m}\right] \leq C\left(1+\mathbb{E}\left[\|u\|_{L^{m}\left((0, T) ; H^{k, m}(D)\right)}^{m}\right]\right), \quad m \geq 2,
$$

and for some $0<\gamma<1 / 2$, that is

$$
z \in L^{m}\left(\Omega ; C^{\theta, \gamma}(\bar{D} \times[0, T])\right) .
$$

Step 4. Higher spatial Hölder regularity of $y$. Next, taking estimate (4.3) as starting point and using Schauder's theory for deterministic parabolic PDEs [41, Theorem 6.48] as well as the linear growth condition on non-local term $F$ we derive

$$
\|y\|_{C^{1+\alpha,(1+\alpha) / 2}(\bar{D} \times[0, T])}^{m} \leq C\left(1+\|\xi\|_{C^{1+l}(\bar{D})}+\|F(u(t))\|_{L^{r}\left((0, T) ; L^{r}(D)\right)}^{r}\right),
$$

for $r \geq 2$ large enough. Hence

$$
y \in L^{m}\left(\Omega ; C^{1+\alpha,(1+\alpha) / 2}(\bar{D} \times[0, T])\right), m \geq 2,
$$

which combined with (4.7) implies

$$
u \in L^{m}\left(\Omega ; C^{1+\beta_{1}, \gamma}(\bar{D} \times[0, T])\right),
$$

with $\beta_{1}=\min \{\theta-1, \alpha\}$.

Step 5. Time regularity. For any $\gamma \in(0,1 / 2)$, due to (4.8), it suffices to improve only the time regularity of $z$. By following the same arguments employed in step 1 for the stochastic integral and using estimate (4.3) we deduce

$$
\mathbb{E}\left[\|z\|_{C^{\gamma}\left([0, T] ; H^{1+k, m}(D)\right)}^{m}\right]<\infty,
$$

which, via the Sobolev embedding $H^{1+k, m}(D) \hookrightarrow C^{1+\beta}(D), \beta<k$, implies that

$$
z \in L^{m}\left(\Omega ; C^{1+\beta_{1}, \gamma}(\bar{D} \times[0, T])\right), m \geq 2 .
$$

Combining now the above estimate with (4.8) we obtain the desired regularity for $u$ and the proof of Theorem 4.1 is complete.

Remark 4.2. For the purposes of the current work the spatial regularity provided by Theorem 4.1 is sufficient. Nevertheless, under the assumption that the drift term $F(u)$ is bounded, which is guaranteed by (3.3) and (4.1), we can get a higher spatial regularity for the solution $u$ of (3.4)-(3.5). In particular, in that case for all $\alpha \in(0,1 / 2)$ there exists $\beta>0$ such that

$$
u \in L^{m}\left(\Omega ; C^{2+\beta, \alpha}(\bar{D} \times[0, T])\right), m \geq 2,
$$


provided also that $\xi \in L^{2}\left(\Omega ; C^{2+l}(\bar{D})\right)$. Indeed, we can increase the spatial regularity of $u$ as long as we consider smoother initial data $\xi$ and smoother non-local terms F. For more details see Propositions 5.2 and 5.3 in [17].

4.2. Strong positivity and Hopf's lemma. According to the approach introduced in [32, the proof of the finite-time blow-up for the deteministic problem (2.1)-(2.3) requires a key estimate of the solution close to the spatial boundary, which is heavily based on Hopf's boundary lemma. For proving a reaction (drift) term induced blow-up for the stochastic problem (1.1)-(1.3) we would like to adjust a similar approach with the deterministic case and thus a Hopf's type lemma in the context of SPDEs should be established.

For readers' convenience we first give a required definition as well as we recall Hopf's maximum principle for deterministic parabolic PDEs, see also [24, 49, 51].

Definition 4.3. ([24]) Let $P_{0}=\left(x_{0}, t_{0}\right)$ be a point on the boundary of $D_{T}$. If there exists a closed ball $B$ centered at $(\bar{x}, \bar{t})$ such that

$$
B \subset \bar{D}_{T}, \quad B \cap \partial D_{T}=\left\{P_{0}\right\}, \bar{x} \neq x_{0},
$$

then we say that $P_{0}$ has the inside strong sphere condition.

Note that the inside strong sphere condition automatically holds when $\Gamma_{T}$ is $C^{2}$.

The following strong positivity result is a key result for proving a Hopf's type lemma.

Theorem 4.4. (Strong positivity) Let $V \equiv V(x, t ; \omega)$ be a weak solution of the following stochastic problem

$$
\begin{aligned}
& \frac{\partial V}{\partial t}=\Delta V+\chi(V)+\sigma(V) \partial_{t} W,(x, t) \in D_{T}, \\
& V(x, t)=0, \quad(x, t) \in \Gamma_{T}, \\
& V(x, 0)=V_{0}(x), \quad x \in D .
\end{aligned}
$$

Let also $\sigma: H \rightarrow L_{2}^{0}(H, H)$ be a Lipschitz continuous function satisfying condition $(\sigma)$ with $\sigma(0)=0$. Assume further that $\chi>0$ with $\chi \in L^{r}\left((0, T) ; L^{r}(D)\right)$ for some $r \geq 2$. In addition we consider initial datum $V_{0}$ which is Holder continuous and satisfies $V_{0}>0$ a.s. in $D$ with $V_{0}=0$ on $\partial D$. Then

$$
\mathbb{P}\{V(x, t)>0 \quad \text { in } \quad D \times[0, T]\}=1,
$$

that is

$$
V>0 \quad \text { a.s. in } D \times[0, T] \text {. }
$$

A proof of Theorem 4.4 can be found either in [45], see Theorem 6.13, or in [14, Theorem 5.1.].

Remark 4.5. Theorem 4.4 actually implies that the solution $V$ of problem (4.10)-(4.12) attains its zero minimum along $\bar{D}_{T}$ only on the boundary $\Gamma_{T}$, also due to the boundary condition (4.11). 
Next we provide a Hopf's lemma for semilinear parabolic SPDEs. In particular, the following holds:

Theorem 4.6. (Hopf's Lemma) Let $V$ be a weak solution of the problem (4.10)-(4.12) where again functions $\chi: H \rightarrow H, \sigma: H \rightarrow L_{2}^{0}(H, H)$ satisfy the same assumptions as in Theorem 4.4. Consider initial condition $V_{0}$ which is Holder continuous and satisfies $V_{0}>0$ a.s. in $D$ with $V_{0}=0$ on $\partial D$. Assume also that $\Gamma_{T}$ is smooth enough, e.g $\Gamma_{T}$ is $C^{2}$, such that it has the inside strong sphere property, then

$$
\left.\frac{\partial V}{\partial \nu}\right|_{P_{0}}<0
$$

for any $P_{0} \in \Gamma_{T}:=\partial D \times(0, T)$. Notably the notion of the derivative into (4.13) should be understood in the classical sence since $V$ is $C^{1}$ with repsect to the spatial variable due to Theorem 4.1 .

Proof. Let $P_{0}=\left(x_{0}, t_{0}\right) \in \Gamma_{T}:=\partial D \times(0, T)$, then since $\Gamma_{T}$ is smooth enough so it has the inside strong sphere property, we can then construct a closed ball $B$ centered at $(\bar{x}, \bar{t}) \neq\left(x_{0}, t_{0}\right)$ and with radius $R$ such that

$$
B \subset \bar{D}_{T}, \quad B \cap \Gamma_{T}=\left\{P_{0}\right\}, \bar{x} \neq x_{0},
$$

i.e., the ball $B$ is tangent to $\Gamma_{T}$ at the point $P_{0}$. Without loss of generality we may assume that the interior of $B$ lies in $D_{T} \cap \mathcal{V}$ for some neighborhood $\mathcal{V}$ of $P_{0}$. We also consider a ball $B_{1}$ centered at $P_{0}$ and of radius $\rho<\left|x_{0}-\bar{x}\right|$, see Fig. 1,

Let $\Gamma_{1}=\partial B_{1} \cap \bar{B}$ and $\Gamma_{2}=\partial B \cap B_{1}$ and let $U_{T}$ be the region enclosed by the curves $\Gamma_{1}$ and $\Gamma_{2}$. Since, by Theorem 4.4, $V>\min _{\bar{D}_{T}} V=0$ a.s. on $\Gamma_{1}$ then we can find $\eta>0$ such that

- (i) $V \geq \eta>0 \quad$ on $\Gamma_{1}$, a.s.

- (ii) $V>0$ on $\Gamma_{2} \backslash\left\{P_{0}\right\}$, a.s. and

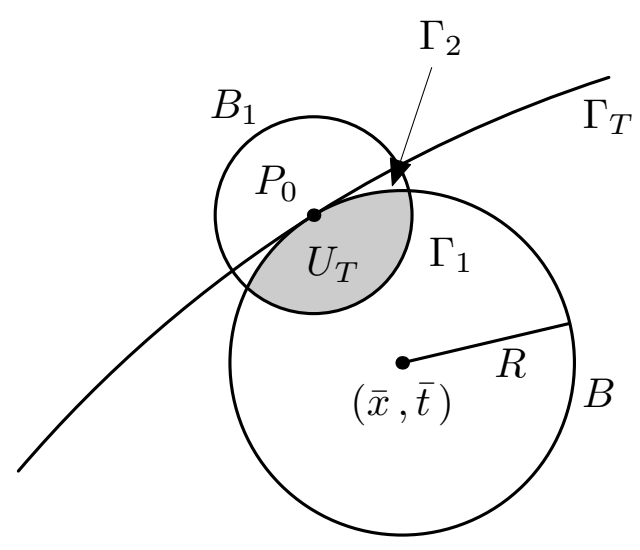

FiguRE 1. The inside strong sphere condition configuration 
- $($ iii $) V\left(P_{0}\right)=0$, a.s.,

see also Fig. 1 .

Consider now the auxiliary deterministic function $h(x, t)$ defined by

$$
h(x, t)=e^{-\alpha R^{2}}-e^{-\alpha\left(|x-\bar{x}|^{2}+(t-\bar{t})^{2}\right)}<0, \quad \alpha>0, \quad \text { for } \quad(x, t) \in U_{T},
$$

Evidently $h=0$ on $\partial B$, and by selecting $\alpha$ sufficiently large we can attain $\mathcal{H}(h)=$ $\frac{\partial h}{\partial t}-\Delta h:=g(x, t)<0$ in $U_{T}$, see also [51]. There also holds

$$
\left.\frac{\partial h}{\partial \nu}\right|_{P_{0}}=2 \alpha R e^{-\alpha R^{2}}>0 .
$$

Let now $\Theta:=V+\varepsilon h, \varepsilon>0$, then, in view of $(i)$, we can find $\varepsilon$ small enough such that $\Theta>0$ on $\Gamma_{1}$ a.s. . Furthermore, by virtue of $(i i)$ and $($ iii $)$, along with the fact that $h=0$ on $\partial B$, we derive $\Theta>0$ a.s. on $\Gamma \backslash\left\{P_{0}\right\}$ and $\Theta\left(P_{0}\right)=0$ a.s. . Note also that $\Theta$ is a weak solution of

$$
\begin{aligned}
& \frac{\partial \Theta}{\partial t}=\Delta \Theta+\widetilde{\chi}(\Theta)+\sigma(\Theta-\varepsilon h) \partial_{t} W, \quad \text { in } U_{T}, \\
& \Theta(x, t)=0, \quad(x, t) \in \partial B \\
& \Theta(x, 0)=\Theta_{0}(x), \quad x \in U_{0},
\end{aligned}
$$

where $\tilde{\chi}(\Theta):=\chi(\Theta-\varepsilon h)+\varepsilon g(x, t)>0$ in $U_{T}$ and $\Theta_{0}(x)>0$ in $U_{0}$ for choosing $\varepsilon$ small enough.

Accordingly, by virtue of Theorem 4.4 we deduce that the minimum of $\Theta$ in $\bar{U}_{T}$ is attained only at $P_{0}$.

Therefore, since by Theorem 4.1 we have that $\Theta$ is $C^{1}$ with respect to the spatial variable on the boundary of $U_{T}$, so we finally deduce

$$
\left.\frac{\partial \Theta}{\partial \nu}\right|_{P_{0}} \leq 0, \quad \text { a.s. }
$$

or equivalently

$$
\frac{\partial V}{\partial \nu}+\varepsilon \frac{\partial h}{\partial \nu} \leq 0, \quad \text { a.s., } \quad \text { at } P_{0}
$$

Therefore, (4.15) in conjunction with (4.14) entails

$$
\left.\frac{\partial V}{\partial \nu}\right|_{P_{0}}<0, \quad \text { a.s. }
$$

and the proof is complete.

Remark 4.7. The result of Theorem 4.6 is still valid if instead of the outward normal direction at $P_{0}$ another outward direction is considered apart from the tangential one. 
4.3. Estimates near the boundary. In order to tackle the difficulties arising from the presence of the non-local term $K(t)=\left(\int_{D} f(u(x, t)) d x\right)^{-q}$, in (1.1)-(1.3) we need to estimate the contribution of $u(x, t)$ near the boundary. For that purpose we will use the moving plane method as in [32], which is actually inspired by the results in the seminal paper by Gidas et al. [25]. Although most of the implemented arguments are quite standard in the context of deterministic PDEs, since it is the first time that those ideas are employed for SPDEs a detailed proof is provided.

Lemma 4.8. Let $u(x, t)$ be the solution of (1.1) $-(1.3)$ with initial data $\xi \in L^{2}\left(\Omega ; L^{\infty}(D)\right)$ satisfying $\xi>0$ a.s. in $D$ with $\xi=0$ on $\partial D$. Assume further that the nonlinearity $f$ is an increasing function as well as $D \subset \mathbb{R}^{d}, d \geq 1$, is convex and smooth enough as in Theorem 4.6. Then there exists $\bar{D}_{0} \varsubsetneqq D$ such that

$$
\int_{D} f(u) d x \leq(\ell+1) \int_{D_{0}} f(u) d x, \quad \text { for all } \quad 0<\widetilde{t}_{0} \leq t<T, \quad \text { a.s. }
$$

where $\ell$ is some positive integer.

Proof. For any $y \in \partial D$ we define the hyperplane

$$
\mathcal{T}(\mu, y):=\left\{x \in \mathbb{R}^{d}:(x, \nu(y))_{d}=\mu\right\},
$$

where $(\cdot, \cdot)_{d}$ stands for the inner product in $\mathbb{R}^{d}$.

Then we can find $\mu_{0}$ such that $\mathcal{T}\left(\mu_{0}, y\right)$ coincides with the tangent hyperplane to $D$ at $y$ and $y \in \mathcal{T}\left(\mu_{0}, y\right) \cap \bar{D}$ (note that when $D$ is strictly convex then $\mathcal{T}\left(\mu_{0}, y\right) \cap \bar{D}=\{y\}$ ), see Fig. 2.

Since $D$ is a bounded set there exists $\mu_{1}<\mu_{0}$ such that $\mathcal{T}(\mu, y) \cap \bar{D}=\emptyset$ for $\mu>\mu_{0}$ and $\mu<\mu_{0}-\mu_{1}$.

We define

$$
\Sigma(\mu, y):=\left\{x \in D: \mu<(x, \nu(y))_{d}<\mu_{0}\right\},
$$

while by $\Sigma^{\prime}(\mu, y)$ we denote the reflection of $\Sigma(\mu, y)$ across $\mathcal{T}(\mu, y)$. Now using the convexity of $D$ we can choose $\bar{\mu}$ sufficiently close to $\mu_{0}$ so that $\Sigma^{\prime}(\bar{\mu}, y) \subset D$, see also Fig. 2 ,

Applying now Theorem 4.6, since all its hypotheses are satisfied (see also Theorem 4.1), we deduce that for any $y \in \partial D$

$$
\frac{\partial u(y, t)}{\partial \nu}=(\nabla u(y, t), \nu(y))_{d}<0, \quad \text { a.s., } \text { for any } t \geq t_{0}>0 .
$$

By the spatial regularity of $u$, see (4.2), we can find a neighbourhood of $y$, say $\mathcal{N}_{y}$, such that

$$
\frac{\partial u\left(x, t_{0}\right)}{\partial \nu}=\left(\nabla u\left(x, t_{0}\right), \nu(y)\right)_{d}<0, \quad \text { a.s., } \quad \text { for any } \quad x \in \mathcal{N}_{y} .
$$

We consider now a coordinate system centered at $y$ and defined by $\left(y ; \nu(y), \mathcal{T}\left(\mu_{0}, y\right)\right)$ such that every $x \in \mathbb{R}^{d}$ is expressed as $x=\left(x_{\nu}, x_{\mathcal{T}}\right)$, where $x_{\nu}$ is the component in the direction of $\nu(y)$ while $x_{\mathcal{T}}$ stands for the component in the direction of the hyperplane $\mathcal{T}\left(\mu_{0}, y\right)$. 
Let us define the cylinder $C_{\delta}(y)=\left\{y=\left(x_{\nu}, x_{\mathcal{T}}\right) \in \mathbb{R}^{d}|| x_{\nu}|<\delta,| x_{\mathcal{T}} \mid<\delta\right\}$. We may pick $\delta>0$ small enough so that the reflection of $\overline{C_{\delta}(y)} \cap D$ across $\mathcal{T}(\bar{\mu}, y)$, denoted by $C_{\delta}^{\prime}(y)$, is compact in $D$.

Set $K_{y}=\mathcal{T}\left(\mu_{0}, y\right) \cap \bar{D}$, then $K_{y}$ is a compact convex set and $K_{y}=\bigcap_{\mu<\mu_{0}} \Sigma(\mu, y)$. Every $\hat{y} \in K_{y}$ has the same exterior normal $\nu(y)$. Then we can define an open neighbourhood of $\hat{y}$ of the shape $C_{\delta}(\hat{y})$ and on which $\left(\nabla u\left(\hat{y}, t_{0}\right), \nu(y)\right)_{d}<0$ almost surely (a.s.). Moreover, $K_{y} \subset \bigcup_{\hat{y}} C_{\delta}(\hat{y})$ and since $K_{y}$ is compact we can extract a finite cover of $C_{\delta}(\hat{y})$, say $E=\bigcup_{i=1}^{n} C_{\delta}\left(\hat{y}_{i}\right)$ which contains $K_{y}$, for some positive integer $n=n(y)$.

Since $D$ is convex we can find $\mu<\mu_{0}$ such that $\Sigma(\mu, y) \subset E$ and $\Sigma^{\prime}\left(\rho_{0}, y\right) \subset$ $D, \Sigma\left(\rho_{0}, y\right) \cup \Sigma^{\prime}\left(\rho_{0}, y\right) \subset E$ for $\rho_{0}=\frac{\mu+\mu_{0}}{2}$. (Note that if $D$ is strictly convex then the above construction is unnecessary).

We now set $z(x, t)=z\left(x_{\nu}, x_{\mathcal{T}}, t\right)=u\left(2 \rho_{0}-x_{\nu}, x_{\mathcal{T}}, t\right)$ for $x \in \Sigma\left(\rho_{0}, y\right)$; actually $z$ is the reflection of $u$ across $T\left(\rho_{0}, y\right)$. Then $z$ is a weak solution of

$$
\begin{aligned}
& \frac{\partial z}{\partial t}=\Delta z+K(t) f(z)+\sigma(z) \partial_{t} W(x, t), \quad \text { on } \quad \Sigma\left(\rho_{0}, y\right) \times\left(\widetilde{t}_{0}, T_{\max }\right) \\
& z \geq u \geq 0 \quad \text { on } \quad K_{1}:=\left(\partial D \cap \Sigma\left(\rho_{0}, y\right)\right) \times\left(\widetilde{t}_{0}, T_{\max }\right), \\
& z=u \quad \text { on } \quad K_{2}:=\left(D \cap \mathcal{T}\left(\rho_{0}, y\right)\right) \times\left(\widetilde{t}_{0}, T_{\max }\right) .
\end{aligned}
$$

Consequently $z$ and $u$ satisfy in a weak form the same $\operatorname{SPDE}$ on $\Sigma\left(\rho_{0}, y\right) \times\left(\widetilde{t}_{0}, T_{\max }\right)$ while $z \geq u$ on $K_{1} \cup K_{2}$ and $z\left(\cdot, t_{0}\right) \geq u\left(\cdot, t_{0}\right)$ on $\Sigma\left(\rho_{0}, y\right)$ almost surely (a.s.), hence by the comparison principle, [10, Section 5.1], we deduce that $z \geq u$ almost surely (a.s.) on $\Sigma\left(\rho_{0}, y\right) \times\left(\widetilde{t_{0}}, T_{\max }\right)$.

Note that $\Sigma\left(\rho_{0}, y\right)$ contains an open set of the type $C_{\delta}(y) \cap D$ and if we choose $\delta<\mu_{0}-\rho_{0}$ then the reflection of $C_{\delta}(y) \cap D$ across $\mathcal{T}\left(\rho_{0}, y\right)$ has a compact closure in $D$. We can repeat the above construction for any $y \in \partial D$ and the collection of all cylinders $\left\{C_{\delta}(y)\right\}_{y \in \partial D}$ builds up an open cover of $\partial D$ from which we can extract a finite subcover denoted by $C_{\delta}\left(y_{1}\right), \ldots, C_{\delta}\left(y_{\ell}\right)$ such that $\partial D \subseteq C_{\delta}\left(y_{1}\right) \cup \ldots \cup C_{\delta}\left(y_{\ell}\right)$.

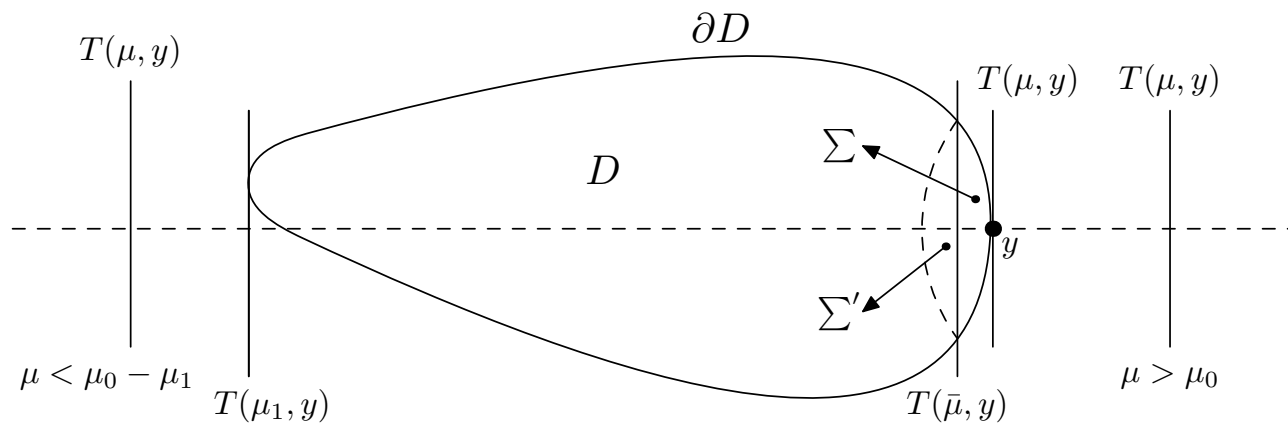

FiguRE 2. The moving plane parallel configuration 
Set $D_{0}=D \backslash \bigcup_{i=1}^{\ell} C_{\delta}\left(y_{i}\right)$, then $\bar{D}_{0} \subset D$ and we have

$$
\begin{aligned}
\int_{D} u d x & \leq \int_{D_{0}} u d x+\sum_{i=1}^{\ell} \int_{C_{\delta}\left(y_{i}\right) \cap D} u d x \leq \int_{D_{0}} u d x+\sum_{i=1}^{\ell} \int_{C_{\delta}\left(y_{i}\right) \cap D} z d x \\
& \leq \int_{D_{0}} u d x+\sum_{i=1}^{\ell} \int_{C_{\delta}^{\prime}\left(y_{i}\right)} z d x=\int_{D_{0}} u d x+\sum_{i=1}^{\ell} \int_{C_{\delta}^{\prime}\left(y_{i}\right)} u d x \\
& \leq \int_{D_{0}} u d x+\ell \int_{D_{0}} u d x \leq(\ell+1) \int_{D_{0}} u d x \quad \text { a.s. },
\end{aligned}
$$

taking also into account that $u \leq z$ on $C_{\delta}\left(y_{i}\right) \cap D$ and $u=z \quad$ on $C_{\delta}^{\prime}\left(y_{i}\right)$ a.s. by reflection.

Now since $f(s)$ is increasing we finally deduce

$$
\int_{D} f(u) d x \leq(\ell+1) \int_{D_{0}} f(u) d x \quad \text { a.s., }
$$

and the proof of lemma is now complete.

4.4. Finite-time blow-up. Henceforth, the nonlinearity $f(s)$ is imposed to satisfy

$$
\left[f^{1-q}(s)\right]^{\prime \prime} \geq 0 \quad \text { for } \quad s \in \mathbb{R} \quad \text { and } \quad \int_{b}^{\infty} \frac{d s}{f^{1-q}(s)}<\infty, \quad \text { for any } b \in \mathbb{R} .
$$

We first prove a blow-up result when the parameter $\lambda$ is large enough.

Theorem 4.9. Suppose that (1.1)-(1.3) has a (unique) local-in-time solution u whose existence is provided by Theorem 3.3 . Assume further that the nonlinearity $f$ satisfies conditions (4.1) and (4.16) as well as $D \subset \mathbb{R}^{d}, d \geq 1$, is convex and smooth enough as in Theorem 4.6. Then u blows up in finite time for sufficiently large values of the parameter $\lambda$, provided that $\xi \in L^{2}(\Omega ; H)$ with $\xi(x)>0$ a.s. in $D$ and $\xi=0$ on $\partial D$.

Proof. Let us define $\widehat{u}(t)$ as in the proof of Theorem 3.8. Now taking the expectation over (3.17) we have

$$
\begin{aligned}
\mathbb{E}[\hat{u}(t)] & =\mathbb{E}\left[\int_{D} \xi(x) \phi_{1}(x) d x\right]-\lambda_{1} \mathbb{E}\left[\int_{0}^{t} \int_{D} u(x, s) \phi_{1}(x) d x d s\right] \\
& +\lambda \mathbb{E}\left[\int_{0}^{t} \int_{D} \frac{f(u(x, s)) \phi_{1}(x)}{\left(\int_{D} f(u(x, s)) d x\right)^{q}} d x d s\right]
\end{aligned}
$$

taking also into account that

$$
\mathbb{E}\left[\int_{0}^{t} \int_{D} \sigma(u(x, s)) \phi_{1}(x) d x d W(s)\right]=0 .
$$

For simplicity, hereafter, we will write $u(x, t)$ and $\phi(x)$ as $u$ and $\phi$, respectively in the integrand. 
Set $\Psi(t)=\mathbb{E}[\hat{u}(t)]$, then by using again Fubini's theorem, we deduce

$$
\Psi(t)=\Psi_{0}-\lambda_{1} \int_{0}^{t} \Psi(s) d s+\lambda \mathbb{E}\left[\int_{0}^{t} \int_{D} \frac{f(u) \phi_{1}}{\left(\int_{D} f(u) d x\right)^{q}} d x d s\right],
$$

where $\Psi_{0}=\mathbb{E}\left[\left(\xi, \phi_{1}\right)_{H}\right]$, or equivalently the initial value problem

$$
\frac{d \Psi}{d t}=-\lambda_{1} \Psi(t)+\lambda \mathbb{E}\left[K(t) \int_{D} f(u) \phi_{1} d x\right], \quad t>0, \quad \Psi(0)=\Psi_{0} .
$$

By Lemma 4.8, we can construct $D_{0} \subset D$ with $\bar{D}_{0} \varsubsetneqq D$ such that

$$
\int_{D} f(u) d x \leq(\ell+1) \int_{D_{0}} f(u) d x, \quad \text { almost surely (a.s.), }
$$

for some $\ell \in \mathbb{N}$. Let $m_{1}=\inf _{x \in D_{0}} \phi_{1}(x)$, then since $\bar{D}_{0} \varsubsetneqq D$ we have $m_{1}>0$. Hence

$$
\int_{D} f(u) d x \leq \frac{\ell+1}{m} \int_{D_{0}} f(u) \phi_{1} d x \leq \frac{\ell+1}{m_{1}} \int_{D} f(u) \phi_{1} d x, \quad \text { almost surely (a.s.), }
$$

and so

$$
K(t)=\left(\int_{D} f(u) d x\right)^{-q} \geq L\left(\int_{D} f(u) \phi_{1} d x\right)^{-q}, \quad \text { almost surely (a.s.), }
$$

for

$$
L=\left(\frac{m_{1}}{\ell+1}\right)^{q}
$$

Therefore by virtue of (4.20) and applying Jensen's inequality twice, since both $f(s)$ and $f^{1-q}(s)$ are convex functions, see also (4.1) and (4.16), we deduce

$$
\begin{aligned}
\mathbb{E}\left[K(t) \int_{D} f(u) \phi_{1} d x\right] & \geq \mathbb{E}\left[L\left(\int_{D} f(u) \phi_{1} d x\right)^{1-q}\right] \\
& \geq L f^{1-q}(\mathbb{E}[\hat{u}(t)])=L f^{1-q}(\Psi(t)) .
\end{aligned}
$$

Thus by virtue of (4.19) and (4.22) the differential inequality holds

$$
\frac{d \Psi(t)}{d t} \geq-\lambda_{1} \Psi(t)+\lambda L f^{1-q}(\Psi(t)), \quad t>0
$$

with initial condition $\Psi(0)=\Psi_{0}$.

Define

$$
0<N:=\sup _{s>\Psi(0)} \frac{s}{f^{1-q}(s)}
$$

then due to (4.16) we have that $N<\infty$, and so choosing $\lambda>\frac{\lambda_{1} N}{L}$, we deduce

$$
t \leq \int_{\Psi(0)}^{\Psi(t)} \frac{d s}{\lambda L f^{1-q}(s)-\lambda_{1} s} \leq \frac{1}{\Lambda} \int_{\Psi(0)}^{\Psi(t)} \frac{d s}{f^{1-q}(s)}<\frac{1}{\Lambda} \int_{\Psi(0)}^{\infty} \frac{d s}{f^{1-q}(s)}<\infty,
$$

for

$$
0<\Lambda \leq \lambda L-\lambda_{1} N<\infty
$$


Thus $\Psi(t)$ blows up in finite time, i.e. $\Psi(t) \rightarrow \infty$ as $t \rightarrow T_{1}$ where $T_{1}$ is estimated as

$$
T_{1} \leq \int_{\Psi(0)}^{\infty} \frac{d s}{\lambda L f^{1-q}(s)-\lambda_{1} s} \leq \frac{1}{\Lambda} \int_{\Psi(0)}^{\infty} \frac{d s}{f^{1-q}(s)}<\infty .
$$

Indeed, since by Theorem $4.1 u$ is bounded in $D \times[0, T)$, then (3.16) yields

$$
\Psi(t)=\mathbb{E}\left[\int_{D} u \phi_{1}(x) d x\right] \leq \mathbb{E}\left[\|u\|_{\infty}\right],
$$

and thus $\mathbb{E}\left[\|u\|_{\infty}\right] \rightarrow \infty$ as $t \rightarrow T^{*} \leq T_{1}$.

Next we prove that blow-up also occurs for large enough initial data.

Theorem 4.10. Suppose that the assumptions of Theorem 4.9 hold true. Assume also that

$$
\mathbb{E}\left[\int_{D} \xi \phi_{1} d x\right]>\zeta
$$

where $\zeta=\zeta(\lambda)$ is the largest root of the equation

$$
\alpha(s):=\lambda L f^{1-q}(s)-\lambda_{1} s=0,
$$

and $L$ is the constant given by (4.21). Then the solution $u$ of (3.4)-(3.5) blows up in finite time.

Proof. Following the same steps as in the proof of Theorem 4.9 we obtain that $\Psi(t)=$ $\mathbb{E}\left[\int_{D} u \phi_{1} d x\right]$ satisfies the differential inequality

$$
\frac{d \Psi(t)}{d t} \geq-\lambda_{1} \Psi(t)+\lambda L f^{1-q}(\Psi(t))=\alpha(\Psi(t)), \quad t>0,
$$

with $\Psi(0)=\Psi_{0}:=\mathbb{E}\left[\int_{D} \xi \phi_{1} d x\right]$.

Let $\zeta=\zeta(\lambda)$ be the largest root of the equation $\alpha(s)=0$. Then by choosing $\Psi_{0}>\zeta$ and using again (4.16) we deduce

$$
t \leq \int_{\Psi_{0}}^{\Psi(t)} \frac{d s}{\alpha(s)} \leq \int_{\Psi_{0}}^{\infty} \frac{d s}{\alpha(s)} \leq \frac{1}{\Lambda_{1}} \int_{\Psi_{0}}^{\infty} \frac{d s}{f^{1-q}(s)}<\infty,
$$

for some positive constant $\Lambda_{1}$. But the above relation entails that $\Psi(t)$ blows up in finite time $T_{1}<\infty$, where

$$
T_{1} \leq \frac{1}{\Lambda_{1}} \int_{\Psi_{0}}^{\infty} \frac{d s}{f^{1-q}(s)}<\infty,
$$

which, similarly to Theorem [4.9, implies that $\mathbb{E}\left[\|u\|_{\infty}\right] \rightarrow \infty$ as $t \rightarrow T^{*} \leq T_{1}$.

Remark 4.11. Theorems 4.9 and 4.10 both imply explosion in terms of the expectation of the $L^{q}$-norm for any $q \geq 1$ as well. Indeed, since $\phi_{1}$ is bounded and continuous on $\bar{D}$ by applying Hölder's inequality for each $q \geq 1$ we derive

$$
\Psi(t) \leq C_{q} \mathbb{E}\left[\left(\int_{D}|u|^{q} d x\right)^{1 / q}\right],
$$


for $C_{q}=\left(\int_{D}\left|\phi_{1}\right|^{r} d x\right)^{1 / r}$ with $r=q /(q-1)$, which actually yields that the expectation of the $L^{q}$-norm explodes in finite time $T_{q} \leq T^{*}$.

4.5. An estimate of the probability of blow-up. In the current subsection we consider the following

$$
\begin{aligned}
& \frac{\partial u}{\partial t}=\Delta u+F(u)+\kappa u d \beta(t),(x, t) \in D_{T}, \\
& u(x, t)=0, \quad(x, t) \in \Gamma_{T}, \\
& u(x, 0)=\xi(x), \quad x \in D,
\end{aligned}
$$

where now $\{\beta(t), t \geq 0\}$ stands for a standard one-dimensional Brownian motion and $\kappa$ is positive constant. Now, for sake of simplicity we fix the parameter $\lambda=1$ and thus

$$
F(u)=\frac{f(u)}{\left(\int_{D} f(u) d x\right)^{q}}, \quad 0<q<1 .
$$

The domain $D \subset \mathbb{R}^{d}, d \geq 1$, is still assumed to be convex as well as $\Gamma_{T}$ is smooth enough so that it has the inside strong sphere property whereas the nonlinearity $f(s)$ satisfies (4.1) and (4.16). Thus, it is easily seen that the above problem satisfies the assumptions of Lemma 4.8 and thus estimate (4.20) is still valid for its solution.

Next we show that the solution $u$ of (4.26)-(4.28) exhibits a finite-time blow-up, induced again by the non-local term, in the sense

$$
\limsup _{t \rightarrow T}\|u(\cdot, t)\|_{\infty}=\infty
$$

and thus under these circumstances a stronger type rather than blow-up in mean $L^{p}$-norm takes place.

For that purpose we employ a different technique than the one in subsection 4.4, which also provides an upper estimate of the probability of blow-up. To this end, we first introduce the auxiliary random function

$$
v(x, t)=e^{-\kappa \beta(t)} u(x, t), \quad(x, t) \in D_{T},
$$

and we follow closely the approach introduced in [20]. In order, to make our paper selfcontained, we present all the required steps in every detail.

By virtue of Itô's Lemma, see [20, Proposition 1], it can be shown that $v(x, t)$ satisfies the following random PDE problem

$$
\begin{aligned}
& \frac{\partial v}{\partial t}=\Delta v-\frac{\kappa^{2}}{2} v+e^{-\kappa \beta(t)} F\left(e^{\kappa \beta(t)} v\right), \quad(x, t) \in D_{T}, \\
& v(x, t)=0, \quad(x, t) \in \Gamma_{T}, \\
& v(x, 0)=\xi(x), \quad x \in D .
\end{aligned}
$$

Notably, (4.30)-(4.32) should be understood trajectorywise and classical results such as existence, uniqueness and positivity of its a solution up to eventual blow-up can be found in [24, Theorem 9, Chapter 7]. Note that the solution $u$ of (4.26)-(4.28) blows up in finite 
time as long as the solution $v$ of (4.30)-(4.32) does so and due to (4.29) both of them blow up simultaneously.

Set

$$
\left(u(t), \phi_{1}\right):=\int_{D} u(x, t) \phi_{1}(x) d x
$$

where again $\phi_{1}$ stands for the first Dirichlet eigenfunction of $-\Delta$ with corresponding eigenvalue $\lambda_{1}>0$, then by Definition 3.1 we have

$$
\left(u(t), \phi_{1}\right)=\left(\xi, \phi_{1}\right)+\int_{0}^{t}\left[\left(u(s), \Delta \phi_{1}\right)+\left(F(u(s)), \phi_{1}\right)\right] d s+\kappa \int_{0}^{t}\left(u(s), \phi_{1}\right) d \beta(s) .
$$

Next by virtue of (4.29) and Itô's formula we derive

$$
e^{-\kappa \beta(t)}=1-k \int_{0}^{t} e^{-\kappa \beta(s)} d \beta(s)+\frac{k^{2}}{2} \int_{0}^{t} e^{-\kappa \beta(s)} d s
$$

or equivalently in differential form

$$
d\left(e^{-\kappa \beta(t)}\right)=-k e^{-\kappa \beta(t)} d \beta(t)+\frac{k^{2}}{2} e^{-\kappa \beta(t)}
$$

Applying now integration by parts formula, see [44, Corollary 7.11, p. 119],

$$
\begin{aligned}
\widetilde{v}(t):=\left(v, \phi_{1}\right) & =\left(\xi, \phi_{1}\right)+\int_{0}^{t} e^{-\kappa \beta(s)} d\left(u(s), \phi_{1}\right) \\
& +\int_{0}^{t}\left(u(s), \phi_{1}\right) d\left(e^{-\kappa \beta(t)}\right)+\left[e^{-\kappa \beta(t)},\left(u(t), \phi_{1}\right)\right],
\end{aligned}
$$

where the last term in the preceding relation is called quadratic variation and is defined as

$$
\left[e^{-\kappa \beta(t)},\left(u(t), \phi_{1}\right)\right]:=-\int_{0}^{t} \kappa^{2} e^{-\kappa \beta(s)}\left(u(s), \phi_{1}\right) d s, \quad \text { for } \quad 0<t<T,
$$

see also [44, Definition 7.6].

Taking now into account (4.33) we finally get

$$
\begin{aligned}
& \widetilde{v}(t):=\left(v, \phi_{1}\right)=\left(\xi, \phi_{1}\right)+\int_{0}^{t} e^{-\kappa \beta(s)}\left[\left(u(s), \Delta \phi_{1}\right)+\left(F(u(s)), \phi_{1}\right)\right] d s \\
& +\kappa \int_{0}^{t} e^{-\kappa \beta(s)}\left(u(s), \phi_{1}\right) d \beta(s)+\int_{0}^{t}\left(u(s), \phi_{1}\right)\left(-\kappa e^{-\kappa \beta(s)} d \beta(s)+\frac{\kappa^{2}}{2} e^{-\kappa \beta(s)} d s\right) \\
& +\left[e^{-\kappa \beta(t)},\left(u(t), \phi_{1}\right)\right],
\end{aligned}
$$

Consequently by virtue of (4.29) we have

$\left(v(t), \phi_{1}\right)=\left(\xi, \phi_{1}\right)+\int_{0}^{t}\left[-\lambda_{1}\left(v(t), \phi_{1}\right)+e^{-\kappa \beta(s)}\left(F\left(e^{\kappa \beta(s)} v(s)\right), \phi_{1}\right)-\frac{\kappa^{2}}{2}\left(v(s), \phi_{1}\right)\right] d s$ 
which by differentiation with respect to time gives

$$
\frac{d \widetilde{v}(t)}{d t}=-\left(\lambda_{1}+\frac{\kappa^{2}}{2}\right) \widetilde{v}(t)+e^{-\kappa \beta(t)}\left(F\left(e^{\kappa \beta(t)} v(t)\right), \phi_{1}\right),
$$

and by virtue of (4.20) entails

$$
\frac{d \widetilde{v}(t)}{d t} \geq-\left(\lambda_{1}+\frac{\kappa^{2}}{2}\right) \widetilde{v}(t)+L e^{-\kappa \beta(t)}\left(f\left(e^{\kappa \beta(t)} v(t)\right), \phi_{1}\right)^{1-q} .
$$

Assuming now that the nonilearity $f(s)$ satisfies the growth condition

$$
f(s) \geq L^{\frac{1}{(q-1)}} s^{\frac{1+\varepsilon}{1-q}} \text { for all } s>0 \text { and some } \varepsilon>0,
$$

then using Jensen's inequality (4.35) we have

$$
\frac{d \widetilde{v}(t)}{d t} \geq-\left(\lambda_{1}+\frac{\kappa^{2}}{2}\right) \widetilde{v}(t)+e^{\varepsilon \kappa \beta(t)}(\widetilde{v}(t))^{1+\varepsilon}, \quad 0<t<T .
$$

Comparing now the solution of (4.37) with the solution of the following Bernoulli's type initial value problem

$$
\frac{d \mathcal{Y}(t)}{d t}=-\left(\lambda_{1}+\frac{\kappa^{2}}{2}\right) \mathcal{Y}(t)+e^{\varepsilon \kappa \beta(t)}(\mathcal{Y}(t))^{1+\varepsilon}, \quad \mathcal{Y}(0)=\left(\xi, \phi_{1}\right):=\xi_{0},
$$

which is given by

$$
\mathcal{Y}(t)=e^{-\left(\lambda_{1}+\frac{\kappa^{2}}{2}\right) t}\left[\xi_{1}^{-\varepsilon}-\varepsilon \int_{0}^{t} e^{-\left(\lambda_{1}+\frac{\kappa^{2}}{2}\right) \varepsilon s+\varepsilon \kappa \beta(s)} d s\right]^{-\frac{1}{\varepsilon}}, \quad 0 \leq t<T_{\mathcal{Y}},
$$

we get that

$$
\widetilde{v}(t) \geq \mathcal{Y}(t) \text { for all } 0 \leq t \leq \min \left\{T, T_{\mathcal{Y}}\right\}
$$

where

$$
T_{\mathcal{Y}}:=\inf \left\{t \geq 0 \mid \int_{0}^{t} e^{-\left(\lambda_{1}+\frac{\kappa^{2}}{2}\right) \varepsilon s+\varepsilon \kappa \beta(s)} d s \geq \frac{1}{\varepsilon} \xi_{1}^{-\varepsilon}\right\}
$$

denotes the maximum existence time of $\mathcal{Y}(t)$. Note that $\mathcal{Y}(t)$ exhibits finite-time blow-up in the event $\left\{T_{\mathcal{Y}}<\infty\right\}$ and due to (4.38) the function

$$
t \longmapsto \int_{D} u(x, t) \phi_{1}(x) d x
$$

explodes in finite time on the event $\left\{T_{\mathcal{Y}}<\infty\right\}$. Furthermore, $T_{\mathcal{Y}}$ is an upper bound of the blow-up time of $\widetilde{v}(t)$ and since

$$
\widetilde{v}(t)=\int_{D} v(x, t) \phi_{1}(x) d x \leq\|v(\cdot, t)\|_{\infty},
$$


it is also an upper bound of blow-up times for $v$ and $u$. We are now ready to provide a lower bound of the probability of blow-up for $v$ and $u$. First, by (4.39) we have

$$
\begin{aligned}
P\left[T_{\mathcal{Y}}=+\infty\right] & =P\left[\int_{0}^{t} \exp \left(-\left(\lambda_{1}+\frac{\kappa^{2}}{2}\right) \varepsilon s+\kappa \varepsilon \beta(s)\right) d s<\frac{1}{\varepsilon} \xi_{1}^{-\varepsilon} \text { for all } t>0\right] \\
& =P\left[\int_{0}^{\infty} \exp \left(-\left(\lambda_{1}+\frac{\kappa^{2}}{2}\right) \varepsilon s+\kappa \varepsilon \beta(s)\right) d s \leq \frac{1}{\varepsilon} \xi_{1}^{-\varepsilon}\right] \\
& =P\left[\int_{0}^{\infty} \exp \left(2 \hat{\varepsilon} \beta(s)^{(\ell)}\right) d s \leq \frac{1}{\varepsilon} \xi_{1}^{-\varepsilon}\right]
\end{aligned}
$$

where $\beta(s)^{(\ell)}:=\ell s+\beta(s), \ell:=-\frac{\left(\lambda_{1}+\frac{\kappa^{2}}{2}\right)}{\kappa}$, and $\hat{\varepsilon}:=\frac{\kappa \varepsilon}{2}$. Using the new time scale $s \mapsto s \cdot \hat{\varepsilon}^{2}$ we finally get

$$
P\left[T_{\mathcal{Y}}=+\infty\right]=P\left[\frac{4}{\kappa^{2} \varepsilon^{2}} \int_{0}^{\infty} \exp \left(2 \beta(s)^{(\widehat{\ell})}\right) d s \leq \frac{1}{\varepsilon} \xi_{1}^{-\varepsilon}\right]
$$

where $\hat{\ell}=\frac{\ell}{\hat{\varepsilon}}$. The distribution of the integral term in (4.41) can be identified by either using some formulas in [8, 19, 54] or otherwise by following the approach in [48] and therefore we obtain

$$
\int_{0}^{\infty} \exp \left(2 \beta(s)^{(\hat{\ell})}\right) d s=\frac{1}{2 Z_{-\hat{\ell}}}
$$

where the above relation should be understood in distributional sense. Here $Z_{-\hat{\ell}}$ is a random variable following the law

$$
P\left(Z_{-\hat{\ell}} \in d y\right)=\frac{1}{\Gamma(-\hat{\ell})} e^{-y} y^{-\hat{\ell}-1} d y
$$

where $\Gamma(\cdot)$ stands for the standard $\Gamma$ - function, cf. [1].

Consequently by (4.41)

$$
P\left[T_{\mathcal{Y}}=+\infty\right]=\int_{0}^{\frac{1}{\varepsilon} \xi_{1}^{-\varepsilon}} h(y) d y
$$

where

$$
h(y)=\frac{\left(\kappa^{2} \varepsilon^{2} y / 2\right)^{\left(2 \lambda_{1}+\kappa^{2}\right) / \kappa^{2} \varepsilon}}{y \Gamma\left(\left(2 \lambda_{1}+\kappa^{2}\right) /\left(\kappa^{2} \varepsilon\right)\right)} \exp \left(-\frac{2}{\kappa^{2} \varepsilon^{2} y}\right),
$$

see also [8, formula 1.10.4(1)], and thus

$$
P\left[T_{\mathcal{Y}}<+\infty\right]=1-P\left[T_{\mathcal{Y}}=+\infty\right]=\int_{\frac{1}{\varepsilon} \xi_{1}^{-\varepsilon}}^{+\infty} h(y) d y
$$

In this manner we have shown the following

Theorem 4.12. Let the domain $D \in \mathbb{R}^{d}, d \geq 1$, be convex and smooth enough as in Theorem 4.6. Assume further that the nonlinearity $f(s)$ satisfies the growth condition 
(4.36). Then the probability that the solution of (4.26)-(4.28) exhibits finite-time blow-up is lower bounded by the quantity $\int_{\frac{1}{\varepsilon} \xi_{1}^{-\varepsilon}}^{+\infty} h(y) d y$.

Remark 4.13. Note that relation (4.42) guarantees that the solution of (4.26)-(4.28) blows up almost surely once a big enough initial datum $\xi(x)$ is considered, i.e. once

$$
\xi_{1}=\int_{D} \xi(x) \phi_{1}(x) \gg 1
$$

which is in agreement with the result of Theorem 4.10 .

Remark 4.14. In case we consider a non-local term of the form

$$
F(u)=\frac{\lambda f(u)}{\left(\int_{D} f(u) d x\right)^{q}}, \quad 0<q<1
$$

then

$$
T_{\mathcal{Y}}:=\inf \left\{t \geq 0 \mid \int_{0}^{t} e^{-\left(\lambda_{1}+\frac{\kappa^{2}}{2}\right) \varepsilon s+\varepsilon \kappa W_{s}} d s \geq \frac{1}{\lambda \varepsilon} \xi_{1}^{-\varepsilon}\right\}
$$

and thus

$$
P\left[T_{\mathcal{Y}}<+\infty\right]=\int_{\frac{1}{\lambda \varepsilon} \xi_{1}^{-\varepsilon}}^{+\infty} h(y) d y .
$$

Therefore the bigger the value of the control parameter $\lambda$ is then the more probable the solution of (4.26)-(4.28) to exhibit finite-time blow-up becomes; the latter is also consistent with the result of Theorem 4.9 .

Remark 4.15. Note that for $\kappa=0$, when problem (4.26)-(4.28) becomes deterministic and thus $u=v$, then by (4.40) we derive that $P\left[T_{\mathcal{Y}}=+\infty\right]=0$ provided that $\xi_{1}>\lambda_{1}^{1 / \varepsilon}$, recovering the probabilistic counterpart of [32, Theorem 2].

Remark 4.16. A global-in-time existence result for problem (4.26)-(4.28) can be derived following the same lines as in [20, Theorem 5] once the non-linearity $f(s)$ is strictly positive, increasing and satisfies a growth condition of the form

$$
f(s) \leq C s^{1+\varepsilon} \quad \text { for all } s>0 \text { and some } C, \varepsilon>0,
$$

since then

$$
F(u)=\frac{f(u)}{\left(\int_{D} f(u) d x\right)^{q}} \leq \widetilde{C} u^{1+\varepsilon} \quad \text { for all } u>0
$$

for $\widetilde{C}=\frac{C}{(|D| f(0))^{q}}$. In that case, a lower bound of the maximum existence time $T>0$ for the solution $u$ of (4.26) -(4.28) can be also derived, see for example [20, Theorem 5]. 
Acknowledgement. The authors would like to thank the anonymous referees for their valuable comments which improved substantially the current manuscript. They would like also to thank Dr. M. Hofmanová for letting them know about the paper [17] as well as Prof. S. Larsson for his stimulating comments which helped improving the presentation of some of the presented results. Finally, special thanks go to Dr. Joe Gildea for helping the authors with the construction of Fig . 1 and Fig. 2.

\section{REFERENCES}

[1] M. Abramowitz and I.A. Stegun, Handbook of mathematical functions with formulas, graphs, and mathematical tables, volume 55. Dover Publications, New York, 1972. 9th Edition.

[2] M. Al-Refai, N.I. Kavallaris \& M.A. HaJjI, Monotone iterative sequences for non-local elliptic problems, European J. Appl. Math. 22 (2011), 533-552.

[3] P. Baras \& L. Cohen, Complete blow-up after $T_{\max }$ for the solution of a semilinear heat equation, J. Funct. Analysis, 71 (1987), 142-174.

[4] J. W. Bebernes \& A. A. LACEy Global existence and finite-time blow-up for a class of nonlocal parabolic problems, Adv. Differential Equations, 2 (1997), 927-953.

[5] J. W. Bebernes \& P. Talaga, Non-local problems modelling shear banding, Comm. Appl. Nonlinear Anal. 3 (1996), 79-103.

[6] J. W. Bebernes, C. Li \& P. Talaga, Single-point blow-up for non-local parabolic problems, Physica D 134 (1999), 48-60.

[7] D. Blömker, Amplitude Equations for Stochastic Partial Differential Equations, Interdisciplinary Mathematical Sciences-Vol. 3, World Scientific Publishing Co. Inc., 2007.

[8] A.N. Borodin, \& P. Salminen, Handbook of Brownian Motion-Facts and Formulae. Second edition. Probability and its Applications. Birkhäuser Verlag, Basel, 2002.

[9] Z. Brzeźniak, On Stochastic convolution in Banach spaces and applications, Stochastics and Stochastic Reports 61 (1997), 245-295.

[10] M. D. Chekroun, E. Park \& R. Temam, The Stampacchia maximum principle for stochastic partial differential equations and applications, J. Diff. Equations, 260 (2016), 2926-2972.

[11] P-L Chow, Stochastic Partial Differential Equations, Second Edition, Chapman and Hall/CRC, 2015.

[12] P-L CHOw, Explosive solutions of stochastic reaction-diffusion equations in mean $L^{p}-n o r m$, J. Diff. Equations, 250 (2011), 2567-2580.

[13] P-L Chow, Unbounded positive solutions of nonlinear parabolic Itô equations, Comm. Stoch. Anal. 3 (2009), 211-222.

[14] D. Conus, M. Joseph \& D. Khoshnevisan , Correlation-length bounds, and estimates for intermittent islands in parabolic SPDEs, Electron. J. Probab. 17 (2012), 1-15. ISSN: 1083-6489 DOI: 10.1214/EJP.v17-2429.

[15] G. Da Prato \& J. Zabczyk, Stochastic Equations in Infinite Dimensions, Cambridge University Press, Cambridge, 1992.

[16] E.B. Davies, Heat Kernels and Spectral Theory, Cambridge University Press, 1989.

[17] A. Debussche, S. De Moor \& M. Hofmanova, A regularity result for quasilinear stochastic partial differential equations of parabolic type, SIAM J. Math. Anal., 47 (2015), 1590-1614.

[18] L. Denis, A. Matoussi \& L. Stoica, Maximum principle for quasi-linear SPDE's on a bounded domain without regularity assumptions, Stoch. Proc. Appl., Vol 123, (2013), 1104-1137.

[19] D. Dufresne, The distribution of a perpetuity, with applications to risk theory and pension funding, Scand. Actuar. J. 9 (1990), 39-79. 
[20] M. Dozzi \& J.A. López-Mimbela, Finite-time blow-up and existence of global positive solutions of a semi-linear SPDE, Stoch. Process. Applications 120 (2010), 767-776.

[21] H. Fujita, On the blowing up of solutions of the Cauchy problem for $u(t)=\Delta u+u^{1+\alpha}$, J. Fac. Sci. Univ. Tokyo, Sec. IA 13 (1966), 109-124.

[22] H. Fujita, On the nonlinear equations $\Delta u+e^{u}=0$ and $\partial v / \partial t=\Delta v+e^{v}$, Bull. Amer. Math. Soc. 75 (1969), 132-135.

[23] M. Foondun, W. Liu \& E. NAne, Some non-existence results for a class of stochastic partial differential equations J. Diff. Equations, 266 (2019), 2575-2596.

[24] A. Friedman, Partial Differential Equations of Parabolic Type, 1983, Prentice-Hall Inc.

[25] B. Gidas, W-M. Ni \& L. Nirenberg, Symmetry and related properties via maximum principle, Comm. Math. Phys. 68 (1979), 209-243.

[26] H. HinRICHSEn, Non-equilibrium critical phenomena and phase transitions into absorbing states, Adv. Phys. 49 (2000), 815-958.

[27] M. Hofanova, Strong solutions of semilinear stochastic partial differential equations, Nonlinear Differ. Equ. Appl. 20 (2013), 757-778.

[28] S. Kaplan, On the growth of solutions of quasilinear parabolic equations, Comm. Pure Appl. Math 16 (1963), 327-330.

[29] N.I. KaVAlLARIS, Explosive solutions of a stochastic non-local reaction-diffusion equation arising in shear band formation, Math. Meth. Appl. Sciences 38 (2015), 3564-3574.

[30] N.I. KAVALlaRIS, Quenching solutions of a stochastic parabolic problem arising in electrostatic MEMS control, Math. Meth. Appl. Sciences, 41 (2018), 1074-1082.

[31] N. I. Kavallaris \& D. E. Tzanetis, Behaviour of a non-local reactive-convective problem with variable velocity in Ohmic heating of food, Nonlocal elliptic and parabolic problems, Banach Center Publ. 66 (2004), 189-198.

[32] N. I. Kavallaris \& D. E. Tzanetis, On the blow-up of a non-local parabolic problem, Appl. Math. Letters 19 (2006), 921-925.

[33] N.I. Kavallaris \& T. Nadzieja, On the blow-up of the non-local thermistor problem, Proc. Edinburgh. Math. Soc. 50 (2007), 389-409.

[34] N.I. Kavallaris \& T. Suzuki, On the finite-time blow-up of a non-local parabolic equation describing chemotaxis, Diff. Int. Equations 20 (2007), 293-308.

[35] N.I. Kavallaris \& T. Suzuki, Non-Local Partial Differential Equations for Engineering and Biology: Mathematical Modeling and Analysis, Mathematics for Industry Vol. 31 Springer Nature 2018.

[36] A. Krzywicki \& T. Nadzieja, Some results concerning the Poisson-Boltzmann equation, Zastosowania Mat. (Appl. Math. (Warsaw)) 21 (1991), 265-272.

[37] A. A. LACEY, Thermal runaway in a non-local problem modelling Ohmic heating. Part I: Model derivation and some special cases, Euro. J. Appl. Math. 6 (1995), 127-144.

[38] A. A. LAcey, Thermal runaway in a non-local problem modelling Ohmic heating. Part II: General proof of blow-up and asymptotics of runaway, Euro. J. Appl. Math. 6 (1995), 201-224.

[39] A.A. Lacey \& D.E. Tzanetis, Global unbounded solutions to a parabolic equation, J. Diff. Equations, 101 (1993), 80-102.

[40] O.A.Ladyzhenskaya, V. A. Solonnikov \& N.N. Ural'Ceva, Linear and Quasilinear Equations of Parabolic Type, Translations of Mathematical Monographs 23, Amer. Math. Soc., Providence, R. I. , 1968 .

[41] G.M. Lieberman, Second Order Parabolic Differential Equations, World Scientific Publishing Co. Inc., River Edge, NJ, 1996.

[42] G. J. Lord, C. E. Powell \& T. Shardlow, An Introduction to Computational Stochastic PDEs, Cambridge University Press, Cambridge, UK, 2014. 
[43] G. Lv \& J. DuAn, Impacts of noise on a class of partial differential equations, J. Diff. Equations, 258 (2015), 2196-2220.

[44] V. MackevičIus, Introduction to Stochastic Analysis: Integrals and Differential Equations, Wiley 2011.

[45] C. Mueller, Some tools and results for parabolic stochastic partial differential equations. A minicourse on stochastic partial differential equations, pp. 111-144, Lecture Notes in Math., 1962, Springer, Berlin, 2009.

[46] J. VAn NeERven \& J. ZHu, A maximal inequality for stochastic convolutions in 2-smooth Banach spaces, Electr. Comm. Probability 16 (2011), 689-705.

[47] W-M. Ni, P. SACKs \& J. TAVAntzIs, On the asymptotic behavior of solutions of certain quasilinear parabolic equations, J. Diff. Equations 35 (1980), 45-54.

[48] C. Pintoux \& N. Privault, A direct solution to the Fokker-Plank equation for exponential Brownian functionals, Anal. Appl. (Singapore) 8 (2010), 287-304.

[49] M. H. Protter \& H. F. Weinberger, Maximum Principles in Differential Equations, 1984, Springer-Verlag.

[50] F. Sagués, J. M. Sancho \& J. García-Ojalvo, Spatio-temporal order out of noise, Rev. Modern Phys. 79 (2007), 829-882.

[51] J. Smoller, Shock Waves and Reaction-Diffusion Equations, 2nd Edition 1983, Springer-Verlag.

[52] D. E. Tzanetis, Blow-up of radially symmetric solutions of a non-local problem modelling ohmic heating, Electron. J. Diff. Eqns. 11 (2002), 1-26.

[53] H. TRIEBEL, Interpolation Theory, Function Spaces, Differential Operators, 2nd Edition 1995, Johann Ambrosius Barth, Heidelberg.

[54] M. Yor, On some exponential functionals of Brownian motion, Adv. Appl. Probab. 24 (1992), 509-531.

[55] G. Wolansky, A critical parabolic estimate and application to non-local equations arising in chemotaxis, Appl. Anal. 66 (1997), 291-321.

Department of Mathematics, University of Chester, Thornton Science Park Pool LAne, InCE, Chester CH2 4NU, UK

E-mail address: n.kavallaris@chester.ac.uk

Department of Mathematics, University of Chester, Thornton Science Park Pool LANE, InCE, Chester CH2 4NU, UK

E-mail address: y.yan@chester.ac.uk 